\title{
Therapeutic Antibodies for the Treatment of Respiratory Tract Infections-Current Overview and Perspectives
}

\author{
Alexie Mayor ${ }^{1,2} \mathbb{D}^{D}$, Adélaïde Chesnay ${ }^{1,2,3}$, Guillaume Desoubeaux ${ }^{1,2,3}$, David Ternant ${ }^{2,4,5,6}$, \\ Nathalie Heuzé-Vourc' ${ }^{1,2}$ (D) and Thomas Sécher ${ }^{1,2, *}$ \\ 1 INSERM, Centre d'Etude des Pathologies Respiratoires, U1100, F-37032 Tours, France; \\ alexie.mayor@univ-tours.fr (A.M.); adelaide.chesnay@univ-tours.fr (A.C.); \\ guillaume.desoubeaux@univ-tours.fr (G.D.); nathalie.vourch@univ-tours.fr (N.H.-V.) \\ 2 Faculty of Medecine, Université de Tours, F-37032 Tours, France; david.ternant@univ-tours.fr \\ 3 CHRU de Tours, Parasitologie-Mycologie-Médecine Tropicale, F-37044 Tours, France \\ 4 CHRU de Tours, Pharmacologie Médicale, Pôle Biologie Médicale, F-37044 Tours, France \\ 5 CHRU de Tours, Centre Pilote de suivi Biologique des Traitements par Anticorps (CePiBAc), \\ F-37044 Tours, France \\ 6 EA 7501 GICC-PATCH, F-37032 Tours, France \\ * Correspondence: thomas.secher@univ-tours.fr; Tel.: +33-(0)-2-4736-6253
}

check for updates

Citation: Mayor, A.; Chesnay, A.; Desoubeaux, G.; Ternant, D.; Heuzé-Vourc'h, N.; Sécher, T. Therapeutic Antibodies for the Treatment of Respiratory Tract Infections-Current Overview and Perspectives. Vaccines 2021, 9, 151. https://doi.org/10.3390/vaccines 9020151

Academic Editor: Tatsuya Yamazaki

Received: 12 January 2021

Accepted: 9 February 2021

Published: 13 February 2021

Publisher's Note: MDPI stays neutral with regard to jurisdictional claims in published maps and institutional affiliations.

Copyright: (c) 2021 by the authors. Licensee MDPI, Basel, Switzerland. This article is an open access article distributed under the terms and conditions of the Creative Commons Attribution (CC BY) license (https:/ / creativecommons.org/licenses/by/ $4.0 /)$.

\begin{abstract}
Respiratorytract infections (RTIs) are frequent and life-threatening diseases, accounting for several millions of deaths worldwide. RTIs implicate microorganisms, including viruses (influenza virus, coronavirus, respiratory syncytial virus (RSV)), bacteria (Pseudomonas aeruginosa, Streptococcus pneumoniae, Staphylococcus aureus and Bacillus anthracis) and fungi (Pneumocystis spp., Aspergillus spp. and very occasionally Candida spp.). The emergence of new pathogens, like the coronavirus SARS-CoV-2, and the substantial increase in drug resistance have highlighted the critical necessity to develop novel anti-infective molecules. In this context, antibodies (Abs) are becoming increasingly important in respiratory medicine and may fulfill the unmet medical needs of RTIs. However, development of Abs for treating infectious diseases is less advanced than for cancer and inflammatory diseases. Currently, only three Abs have been marketed for RTIs, namely, against pulmonary anthrax and RSV infection, while several clinical and preclinical studies are in progress. This article gives an overview of the advances in the use of Abs for the treatment of RTIs, based on the analysis of clinical studies in this field. It describes the $\mathrm{Ab}$ structure, function and pharmacokinetics, and discusses the opportunities offered by the various $\mathrm{Ab}$ formats, $\mathrm{Ab}$ engineering and co-treatment strategies. Including the most recent literature, it finally highlights the strengths, weaknesses and likely future trends of a novel anti-RTI Ab armamentarium.
\end{abstract}

Keywords: therapeutic antibodies; respiratory tract infection; pharmacodynamics (PD); pharmacokinetics (PK); clinical development

\section{Introduction}

Respiratory tract infections (RTI) constitute the third leading cause of morbidity and mortality worldwide, in both children and adults, accounting for approximately 4.25 million deaths per year [1,2]. In addition to premature mortality, RTIs have a dramatic impact on society with respect to disability, healthcare costs and loss of productivity. Thus, the prevention and control of RTIs constitute a major goal of public health.

RTIs refer to a range of infections confined to the upper respiratory tract (rhinitis, sinusitis, pharyngitis or tracheitis) and/or lower respiratory tract (mainly bronchitis and pneumonia), implicating microorganisms, including viruses, bacteria and fungi. According to the World Health Organization (WHO), pneumonia is more lethal in children than the combined mortality from measles, malaria and acquired immune deficiency syndrome (AIDS) [3,4]. 
After more than sixty years, characterized by the use and misuse of broad-spectrum antibiotics, resistance to antibiotics and some antivirals has become a real threat, while thedeficit of drugs with novel modes of action to implement the conventional armamentarium represents a critical concern. The attrition of existing therapeutic options and emergence of new viruses has complicated the management of RTIs, increasing pressure on healthcare systems and motivated the development of new therapeutic strategies for RTIs. Among these, antibodies (Abs), and antibody-based molecules, are now considered as a viable option against emerging viral pathogens and antibiotic-resistant bacteria [5-9].

The pioneer work of Von Behring regarding a diphtheria anti-toxin serum therapy paved the way for the current use of Abs in infectious diseases. Later on, Cecil and Larsen were among the first to conduct a case-control group study to test the efficacy of polyclonal antibody against Streptococcus pneumoniae. Although the success of this study was modest, it allowed to concretely consider Ab therapy in RTIs [10]. Abs present potential advantages compared to other antimicrobials. First, they provide a therapeutic option when vaccines or conventional drugs are neither available nor efficacious, due to compromised immune system or intrinsic resistance. Secondly, Abs usually target highly conserved, specific antigens and are associated with limited development of drug resistance. Finally, in immunocompetent patients, they may provide a prophylactic setting, acting as vaccine-like molecules through the promotion of long-term anti-microbial specific immune responses [11].

Despite extensive investments in Ab discovery and manufacturing, there are currently only three therapeutic Abs approved by the health authorities for fighting against RTIs (Table 1), and fewer than twenty in clinical development. This highlights the complexity of anti-infective $\mathrm{Ab}$ development, especially in the field of infectious diseases, which needs to overcome scientific, regulatory and even commercial barriers before being accessible to patients.

Table 1. Approved therapeutic antibodies for respiratory infections.

\begin{tabular}{ccccc}
\hline Indication & Generic Name & Sponsoring Company & Target & Development Stage \\
\hline Respiratory syncytial virus & Palivizumab (synagis) & AstraZeneca & F-protein & Approved in 1998 \\
Bacillus anthracis & Raxibacumab (abthrax) & GSK & PA & Approved in 2013 \\
& Obiltoxaximab (anthim) & Elusys Therapeutics & PA & Approved in 2016 \\
\hline
\end{tabular}

PA, protective antigen.

In this review, we described the pharmacodynamics (PD) and pharmacokinetic (PK) features of all anti-infectious monoclonal Abs against RTIs that either have been approved by regulatory agencies or are in clinical development for RTIs, and outlined their strengths and limitations. Considering the relevance of Abs to benefit respiratory infections due to emerging pathogens [12], we also highlighted the Abs developed to fight the SARS-CoV-2 pandemic.

\section{Methods}

We undertook a search on Clinical.Trials.gov regarding trials for recombinant monoclonal antibodies in respiratory infectious diseases referenced up to January 1,2021 . The term used in different combinations were as follows: "therapeutic antibodies", "monoclonal antibodies" and pathogen-specific terms (including: Bacillus anthracis, Klebsiella pneumoniae, Mycoplasma pneumoniae, Streptococcus pyogenes, Streptococcus pneumoniae, Group A Streptococcus, Chlamydia pneumoniae, Chlamydia trachomatis, Chlamydia psittaci, Moraxella catarrhalis, Haemophilus influenza, Bordetella pertussis, Coxiella burnetii, Legionella pneumophila, Mycobacterium tuberculosis, Corynebacterium diphtheriae, Neisseria gonorrhoeae, Neisseria meningitis, Francisellatularensis, Xanthomonas pseudomallei, Yersinia pestis, Escherichia coli, Enterobacter, Proteus, Aspergillus spp., Pneumocystis jirovecii, Cryptococcus spp., Candida spp., Coccidioides immitis, Blastomyces dermatitidis, Paracoccidioides brasiliensis, Paragonimus spp., Histoplasma capsulatum, Respiratory syncytial virus, Influenza A virus, Influenza B virus, Hu- 
man papillomavirus, Rhinovirus, Adenovirus, SARS-CoV-2 and COVID-19). The results were limited to on-going trials or trials involving entities for which development is still active (survey of the sponsoring company pipeline). They were confirmed on several drug databases (https://www.antibodysociety.org; https://adisinsight.springer.com/; https: / / www.covid-trials.org/. Our methodology was further documented by specific searches on Pubmed.

\section{Result}

\subsection{Therapeutic Antibodies-Main Features}

Since the development of the hybridoma technology by Köhler and Milstein, in the early 1970s [13] — who earned the Nobel Prize for this work-Ab and Ab-derived molecules have emerged as anti-infective options, even for emerging viral infections [5,14]. Numerous technological advances have been done to improve the efficacy of Abs. From the first-generation murine $\mathrm{Ab}$, innovative approaches have given rise to chimeric, humanized and fully human Abs, which can be sequenced, engineered and bio-produced at a large scale $[15,16]$. The present review will focus on the immunoglobulin $G$ (IgG) scaffold since most Abs approved or under investigation in RTI are full-length IgG. IgG contain an antigen-binding fragment $(\mathrm{Fab})$ recognizing a targeted antigen with a high specificity and affinity, hindering any side effect on symbiotic microflora. They also comprise a fragment crystallizable $(\mathrm{Fc})$ region, mediating the downstream effectors function through its interaction with immune cells (via the engagement with various Fc receptors) or the complement component C1q. The Fc domain (via the engagement with the neonatal receptor for IgG, FcRn) is also intricately associated with a favorable PK, theoretically conferring to IgG a $\approx 21$ day-long half-life (T1/2) in biological fluids [17]. The mechanisms of action of $\mathrm{Ab}$ are multiple and attributable to their Fab region, their Fc region or both. They include direct-killing, neutralization, virulence inhibition, complement deposition, opsonophagocytosis, antibody-dependent cell-mediated cytotoxicity (ADCC) or complement-dependent cytotoxicity (CDC) [14,17].

The structural and functional properties of the natural human IgG subclasses-IgG1, IgG2, IgG3 and IgG4 - can be used to generate therapeutic Abs [18]. Indeed, subclasses differ with regard to molecular structure, PD and PK profiles. However, most Abs on the market are from the IgG1 subclass, most likely because of the stability of IgG1 during the manufacturing process, the greater potency of the effector functions and serum bioavailability [19].

In addition to a multi-faceted $\mathrm{PD}$, the $\mathrm{Ab} \mathrm{PK}$ is unique, involving specific mechanisms of absorption, catabolism and elimination. The fate of the Ab depends on multiple parameters, thereby their PK displays a large inter-individual variability, which can be detrimental for clinical responses [20]. Understanding the parameters governing the $\mathrm{Ab}$ PK better remains critical to enhance the likelihood of an Ab response during RTI.

\subsection{Therapeutic Antibodies against Virus Diseases}

\subsubsection{Respiratory Syncytial Virus (RSV)}

Human respiratory syncytial virus (RSV) belongs to the Paramyxoviridae family. It is an enveloped, single-stranded, negative-sense RNA virus. Its enveloped lipid bilayer contains three membrane proteins: The small hydrophobic protein $(\mathrm{SH})$, the attachment glycoprotein $(G)$ and the fusion protein $(F)[21,22]$. It is noteworthy that the approved and under evaluation (even discontinued) Abs indicated for RSV infection are all directed against the F protein. Indeed, the F protein has an essential role in RSV pathophysiology: The virus binds to host cell using the $G$ protein, and thereafter uses the $F$ protein to fuse with the host cell membranes. As compared to $G$ protein, F protein exhibits a more conserved sequence among the RSV strains and is more immunogenic [23] (Table 2). It is noteworthy that F proteins exist in two forms - the prefusion (pre-F) and post-fusion (post-F) conformations-which may impact antibody binding [23]. 
Table 2. Therapeutic antibodies for viral respiratory infections.

\begin{tabular}{|c|c|c|c|c|c|c|c|}
\hline Indication. & Generic Name & $\begin{array}{l}\text { Sponsoring } \\
\text { Company }\end{array}$ & Target & $\begin{array}{l}\text { Development } \\
\text { Stage }\end{array}$ & NCT & $\begin{array}{c}\text { Completion } \\
\text { Date }\end{array}$ & Status \\
\hline \multirow{2}{*}{$\begin{array}{c}\text { Respiratory } \\
\text { syncytial virus }\end{array}$} & $\begin{array}{l}\text { MEDI-8897 } \\
\text { (nirsevimab) }\end{array}$ & MedImmune & F-protein & Phase $2 / 3$ & $\begin{array}{l}\text { NCT03959488, } \\
\text { NCT03979313 }\end{array}$ & 2021-2023 & Recruiting \\
\hline & MK-1654 & Merck & F-protein & Phase 2 & NCT03524118 & 2022 & Active \\
\hline \multirow{3}{*}{ Influenza $A$ virus } & VIR-2482 & $\begin{array}{c}\text { Vir } \\
\text { Biotechnology }\end{array}$ & HA & Phase 2 & NCT04033406 & 2022 & Recruiting \\
\hline & $\begin{array}{l}\text { CT-P27 } \\
\text { (antibody } \\
\text { mixture) }\end{array}$ & Celltrion & HA & Phase 2 & NCT03511066 & 2018 & $\begin{array}{c}\text { Active on } \\
\text { Celltrion website }\end{array}$ \\
\hline & VIS-410 & Visterra & HA & Phase 2 & $\begin{array}{l}\text { NCT03040141, } \\
\text { NCT02989194, } \\
\text { NCT02468115 }\end{array}$ & 2018 & $\begin{array}{c}\text { Active on } \\
\text { Visterra website }\end{array}$ \\
\hline \multirow{2}{*}{$\begin{array}{c}H P V \text {-associated } \\
\text { Recurrent Respiratory } \\
\text { Papilloma }\end{array}$} & Avelumab & Merck & PD-L1 & Phase 2 & NCT02859454 & 2019 & \multirow{2}{*}{$\begin{array}{c}\text { Active } \\
\text { Active, not } \\
\text { yet recruiting }\end{array}$} \\
\hline & Pembrolizumab & Merck & PD-1 & Phase 2 & NCT02632344 & 2020 & \\
\hline
\end{tabular}

HA (hemagglutinin), PD1 (programmed death 1) and PDL1 (programmed deathligand 1) are responsible for infections, usually limited to the upper airways, including low-febrile rhino-pharyngitis; but, it can spread deeper into the respiratory tract, leading to bronchiolitis or pneumonia. Sensitivity to RSV infection is person-dependent, but mostly involve critical cases at the extremes ages of life and especially in young children during the first two years of life; indeed, a large majority of children contracts RSV and up to $40 \%$ developed a lower respiratory tract infection [24,25]. Despite the health care burden associated with the RSV, there is no curative antiviral drug (except the controversial ribavirin) or vaccine. The main treatment strategy relies on supportive care to relief nasal congestion, fever, dehydration or hypoxia.

Additionally, for supportive care, palivizumab (Synagis ${ }^{\circledR}$ ) was approved in 1998 for prophylaxis of respiratory disease caused by RSV in children at high risk. This humanized IgG1 Ab is the only approved drug targeting specifically the RSV, more precisely, an epitope present both in the pre-F and post-F conformations. Efficacy was demonstrated among children with bronchopulmonary dysplasia, with congenital heart disease or premature infants (Impact-RSV Trial).

Besides, two additional anti-RSV Abs are currently in clinical development: MEDI8897 (nirsevimab) is a fully human IgG1 Ab developed by AstraZeneca for the prevention of RSV infection [26] (Table 2). Compared to palivizumab, MEDI-8897 is 9-fold more effective in reducing viral load in cotton rats. This improved efficacy could be partly explained by the specific targeting of the pre-F conformation. Indeed, it has been observed that the vast majority of potent neutralizing antibodies isolated from the serum of convalescent patients exclusively recognize the pre-F form, which has led to the development of pre-F-specific antibodies as novel therapeutic approaches for RSV infection. It presents also an extended $\mathrm{T} 1 / 2$ (63-73 days in child) consecutive to the introduction of an YTE modification in the Fc fragment. In a Phase 1b/2a (NCT02290340) trial, anti-drug antibodies (ADA) were detected, but were not associated with adverse effects nor a reduction in blood Ab concentration, for up to 151 days. Based on a Phase 3 clinical trial (NCT03979313), MEDI-8897 is expected to improve the prevention against RSV infection in late premature and healthy full-term children. MK-1654 is a fully human Ab-targeting RSV F protein, developed by Merck. It also contains an YTE mutation to increase its serum T1/2 [27]. An interim report from a Phase 1 study (NCT03524118) helped to characterize a PK model (developed for adults), able to predict PK in children and supports further development in children [27].

\subsubsection{Human Papillomavirus (HPV)}

HPV, a well-known driver of genital tract lesions and cervical cancer, may also be responsible for recurrent respiratory papillomatosis (RRP). RRP is characterized by a papilloma growing in the respiratory tract, and can lead to voice changes, pulmonary 
lesions, airway compromise and fatal distal airway obstruction [28]. In 2018, Ahn et al. stained and scored by automated cell count 39 formalin-fixed, paraffin-embedded RPP for CD4, CD8, FoxP3 and PD-1 [29]. They showed that most RRP specimens demonstrated PD-1 (programmed cell death-1) T-lymphocyte infiltration and PD-L1 (programmed deathligand 1) expression on both the papilloma and infiltrating immune cells, suggesting that this checkpoint pathway may contribute to local immunosuppression in RRP. Two clinical trials are in progress to determine whether re-purposed immunotherapies targeting the PD-L1 and PD-1 checkpoint pathway, originally developed to treat cancer patients, can be used to activate the immune system against HPV-infected cells (Table 2). Avelumab is a human IgG1 that binds to PD-L1 and blocks the interaction between PD-L1 and its receptors, PD-1 and B7.1. This leads to the suppression of the inhibitory effects of PD-L1 on cytotoxic $\mathrm{CD} 8+\mathrm{T}$ cells, thus restoring the anti-tumor responses of the T lymphocytes. Avelumab also induces direct lysis of tumor cells by natural killer cells via ADCC [30]. A Phase 2 study (NCT02859454) was conducted to investigate the clinical activity and safety of avelumab in patients with RRP in a two-stage design, with initial enrollment of 12 patients and expansion to 37 patients, if one or more complete response(s) is/are observed in the initial group. The initial enrollment was achieved in 2019 [31]. All patients with laryngeal RRP experienced improvement in disease burden after treatment with avelumab, while no response was observed in patients suffering from pulmonary RRP. Some of the responders developed HPV-specific reactivity in papilloma-infiltrating T-cells that correlated with a reduced viral load. Pembrolizumab, a humanized IgG4k directed against PD-1, prevents the interaction of PD- 1 with its two known ligands PDL-1 and PDL-2 present on tumor cells [32]. In a Phase 2 study (NCT02632344), the investigators are looking at whether pembrolizumab may restore the natural ability of the immune system to recognize and eliminate HPV-infected cells.

\subsubsection{Influenza Virus}

Influenza viruses are negative-sense, single-strand RNA viruses belonging to $\mathrm{Or}$ thomyxoviridae family. There are three types of human influenza viruses- $\mathrm{A}, \mathrm{B}$ and $\mathrm{C}-$ defined according to the expression of nucleoprotein (NP) and matrix (M) protein. Type $A$ and, to a lesser extent, type B are the most frequently encountered in the human population. Their envelope displays two main surface proteins called hemagglutinin (HA) and neuraminidase (NA). HA is implicated in virus entry in host cells via binding to sialic acid, preferentially in the upper airways. NA is involved in the release of the newly produced virus particles. Influenza A viruses are classified into subtypes based on HA (H1-16) and NA (N1-9) antigenic differences. Influenza viruses are responsible for seasonal influenza, which is typically limited to the upper airways and generally leads to mild symptoms, including fever, sneezing, sore throat, coughing, headaches, myalgia and asthenia. However, influenza can eventually be severe when associated with secondary bacterial infection, culminating in devastating pneumonia, especially in children, elderly and chronically disabled persons. Influenza causes 290,000 to 650,000 deaths per year worldwide, with a mortality rate estimated to be $0.2 \%$ [33]. For preventive approaches, inactivated, live attenuated or recombinant HA vaccines are available [34]. However, small mutations in HA and NA, referred to as antigenic drift, frequently occur during each epidemic season and lead to influenza evasion from immune responses. Consequently, the vaccine composition is adjusted and renewed every year. Beside these small changes, major genetic recombination may occur, giving rise to viruses with entirely novel HA or NA, responsible for pandemic flus such as influenza A/H1N1 in 2009. Thus, curative treatments are also necessary. Two main strategies have been considered: (i) to limit the spread of the virus with an NA inhibitor (oseltamivir, peramivir, zanamivir and laninamivir) or M2 ion-channel inhibitor (amantadine and rimantadine); and (ii) to block the viral replication with polymerase acidic (PA) endonuclease inhibitors (baloxavirmarboxil) [35].

However, the emergence of resistant viruses increases the need for new treatments and the interest for anti-influenza Abs. All Abs currently in clinical development target 
HA, considering its essential role on the virus lifecycle. It should also be noted that all Abs are directed against influenza A viruses (Table 2). VIS-410 is a fully human IgG1Ab (Visterra) binding to the stem region of HA, to neutralize the virus. VIS-410 appeared to be well tolerated up to a dose of $50 \mathrm{mg} / \mathrm{kg}$ (NCT02045472) [36]. In a Phase 2 placebocontrolled trial, VIS410 demonstrated a significant anti-influenza activity (NCT02989194, NCT02468115) [37,38]. The results of a study comparing VIS410 in association with oseltamivir to oseltamivir alone (NCT03040141) showed that addition of VIS410 induced a decrease inmortality and faster virus clearance [39]. Celltrion has developed CT-P27, containing two monoclonal Abs directed against influenza HA. In vivo and in vitro studies demonstrated its efficacy against several influenza A subtypes and a Phase 1 trial (KCT000161) showed positive safety results. A Phase 2 trial (NCT02071914) is ongoing. VIR-2482 (VIR Biotechnology) is an engineered $\mathrm{Ab}$ targeting a conserved region of HA. It is intended for flu prophylaxis, since it has a longT1/2. A Phase $1 /$ Phase 2 (NCT04033406) study was launched in 2019 to assess its safety, pharmacokinetics, immunogenicity and efficacy.

\subsubsection{The Severe Acute Respiratory Syndrome Coronavirus 2 (SARS-CoV-2) Pandemic}

SARS-CoV-2 is a Betacoronavirus, like Severe Acute Respiratory Syndrome Coronavirus (SARS-CoV) and Middle East Respiratory Syndrome Coronavirus (MERS-CoV), which are positive-sense, single-stranded RNA viruses. It consists of four major structural proteins: spike glycoproteins (S), forming spikes that hoop from the virus surface; membrane glycoproteins $(\mathrm{M})$; envelope proteins $(\mathrm{E})$, which are transmembrane; and hemagglutinin-esterase dimer protein (HE), containing acetyl-esterase activity; it has a $30 \mathrm{~Kb}$ RNA genome. Viral entry in host cells is achieved by exploiting the link with membrane-bound angiotensin converting enzyme II (ACE2) protein, which is expressed in different pulmonary cell types (type II alveolar cells, upper airways cells) and across the human body (e.g., endothelial cells, kidney or ileum epithelial cells). However, macrophages may also be infected by the SARS-CoV-2 [40]. This probably participates in the extrapulmonary complications associated with SARS-CoV-2. However, pulmonary infection caused by SARS-CoV-2 is the most life-threatening, resulting in some patients in an acute respiratory distress syndrome (ARDS), characterized by an impaired innate immunity, inadequate adaptive immune response, uncontrolled virus- and inflammation-induced tissue damage and vascular permeability (Figure 1) [41]. Much of our understanding of SARS-CoV-2 immunity comes from the incredible amount of studies that has been done during the pandemic and comparison with SARS-CoV/MERS-CoV. Nonetheless, the pathogenesis of COVID-19 remains to be fully elucidated. Briefly, binding of S glycoprotein to ACE2 initiates SARS-CoV-2 entry into host cells. Fusion of the viral and cellular membranes mainly uses the serine protease TMPRSS2 (transmembrane serine protease 2) and endosomal cysteine proteases (cathepsin $\mathrm{B}$ and L). Once SARS-CoV-2 RNA is liberated in the host cell, it is translated and replicatedlike an mRNA - using the host cell machinery. After packaging, the neo-virus is released extracellularly by budding, exocytosis and host cell death. SARS-CoV-2 is a cytopathic virus causing direct cell death during viral replication, resulting in tissue damage and an increased inflammatory response. SARS-CoV-2 replication has been shown throughout the respiratory tract.

The immune response to the virus infection usually starts at the cellular level, and plays a critical role in COVID-19 pathogenesis [42]. On one hand, SARS-CoV-2 will induce antiviral immune responses - both innate and adaptive immune responses - with protective features to control viral infection. It may start in the endosomal compartment of infected host cells, through signaling of pattern-recognition receptors, such as Toll-like receptor 3, which will elicit production of inflammatory cytokines and, in turn, activate the immune cells. Innate immune cells, such as dendritic cells, macrophages and neutrophils, can be activated by cytokines and damage-associated molecular patterns (DAMPs), and initiate adaptive immunity responses. On the other hand, SARS-CoV-2 may elicit pathogenic immune responses. After virus entry into cells, membrane ACE2 is downregulated, lead- 
ing to an imbalance in the renin-angiotensin pathway, thereby promoting angiotensin II-induced vascular permeability and pneumonia with vascular injury. Moreover, activation of Type 1 Angiotensin II Receptor directly upregulates NF-kB and ADAM17, ultimately leading to uncontrolled production of TNF- $\alpha$ and IL-6 [43]. During COVID-19 pathogenesis, activated alveolar macrophages and lung epithelial cells are the major producer of these mediators [44], which, in turn, will at a systemic level mediate emergency myelopoiesis and locally increase the expression of cell adhesion molecules and VEGF [41]. The resulting increase in lung vascular permeability will allow viral dissemination as well as leukocyte infiltration, perpetuating local inflammation, leading to ARDS (Figure 1).

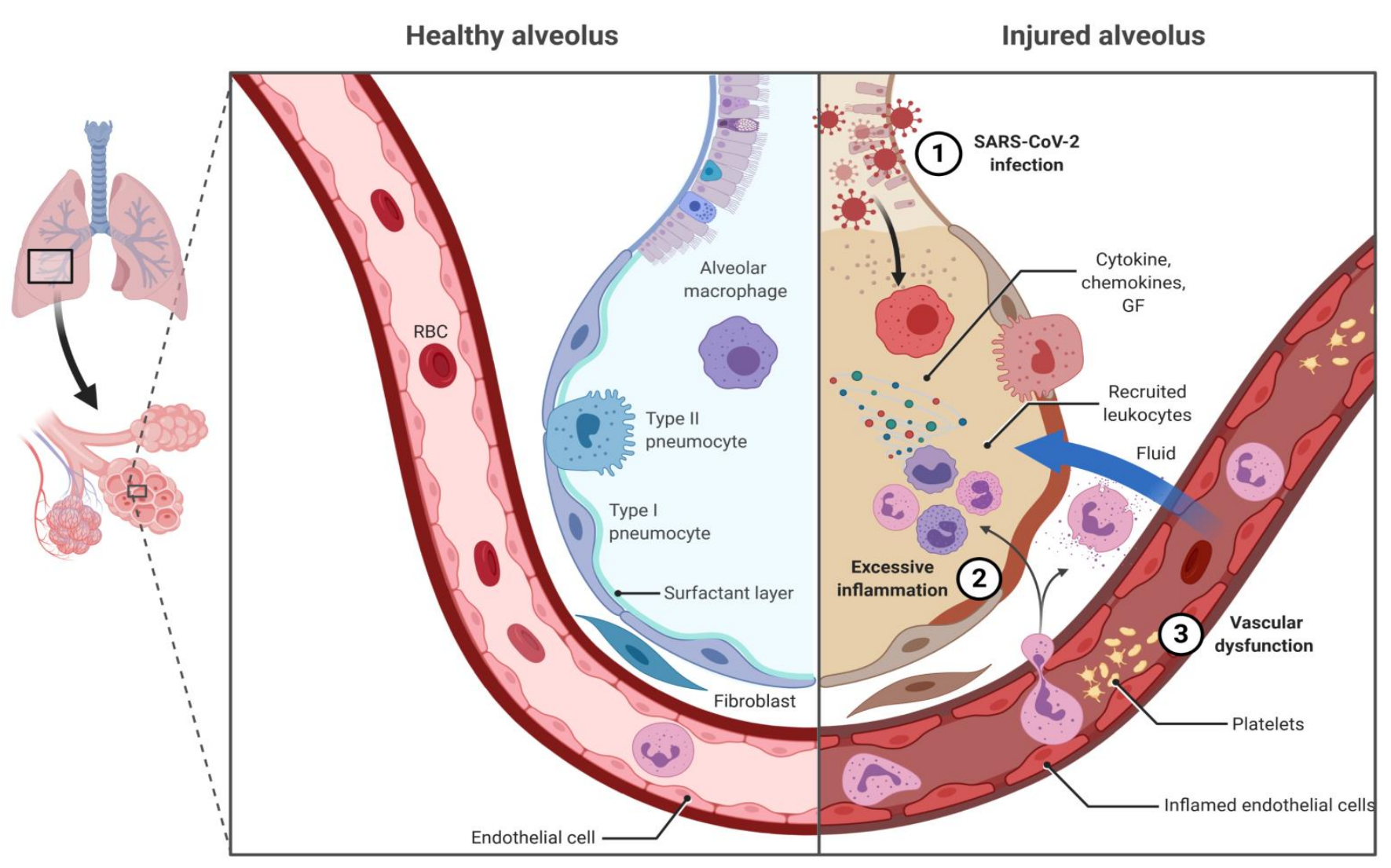

Figure 1. This figure compares the alveolar immune responses in healthy and injured lung after SARS-CoV-2 infection. (1) Coronavirus infects lung cells and encounter alveolar macrophages. (2) Alveolar macrophages identify the virus and initiate innate and acquire immune responses. Cytokines, chemokines or growth factors will attract additional immune responses, which will amplify and perpetuate the inflammation, damaging lung alveoli and endothelium. (3) Endothelium/platelet activation and increased vascular permeability will ultimately provoke vascular dysfunction, allowing virus dissemination and boost leukocytes infiltration.

Over the past few months, many Abs have been developed to face the SARS-CoV-2 pandemic [41,43-45]. We can distinguish two categories of anti-COVID-19 Abs. The first category of anti-COVID-19 Abs is intended to reduce the symptoms and comprise mostly re-purposed Abs, targeting excessive inflammation. The second category includes Abs or Ab-based therapeutics directly targeting SARS-CoV2 (Table 3). 
Table 3. Therapeutic antibodies for the COVID-19 pandemic.

\begin{tabular}{|c|c|c|c|c|c|c|}
\hline Strategy & Generic Name & $\begin{array}{l}\text { Sponsoring } \\
\text { Company }\end{array}$ & Target & $\begin{array}{l}\text { Development } \\
\text { Stage }\end{array}$ & NCT (Status) & $\begin{array}{l}\text { Completion } \\
\text { Date }\end{array}$ \\
\hline \multirow{32}{*}{$\begin{array}{l}\text { ARDS- } \\
\text { related } \\
\text { target }\end{array}$} & IC14 & $\begin{array}{l}\text { Implicit } \\
\text { Bioscience }\end{array}$ & CD14 & Phase 2 & NCT04391309 (Active, not recruiting) & 2021 \\
\hline & Itolizumab & Biocon & CD6 & Phase 2 & NCT04475588 (Completed) & 2020 \\
\hline & Leronlimab & CytoDyn & CCR5 & Phase 2 & $\begin{array}{l}\text { NCT04343651 (Active, not recruiting), } \\
\text { NCT04347239 (Recruiting) }\end{array}$ & 2021 \\
\hline & CPI-006 & $\begin{array}{c}\text { Corvus } \\
\text { Pharmaceuticals }\end{array}$ & CD73 & Phase 1 & NCT04464395 (Recruiting) & 2021 \\
\hline & \multirow{3}{*}{ Meplazumab } & \multirow{3}{*}{$\begin{array}{l}\text { Jiangsu Pacific } \\
\text { Meinuoke Bio } \\
\text { Pharmaceutical }\end{array}$} & \multirow{3}{*}{ CD147 } & Phase 1 & NCT04369586 (Recruiting) & 2020 \\
\hline & & & & Phase $1 / 2$ & NCT04275245 (Recruiting) & 2020 \\
\hline & & & & Phase $2 / 3$ & NCT04586153 (Not yet recruiting) & 2021 \\
\hline & \multirow{2}{*}{ Lanadelumab } & \multirow{2}{*}{ Takeda } & \multirow{2}{*}{ Kallikrein } & Phase $1 / 2$ & NCT04422509 (Recruiting) & 2021 \\
\hline & & & & Phase 1 & NCT04460105 (Withdrawn) & 2020 \\
\hline & Otilimab & GlaxoSmithKline & GM-CSF & Phase 2 & NCT04376684 (Recruiting) & 2020 \\
\hline & \multirow[b]{2}{*}{ Lenzilumab } & \multirow[b]{2}{*}{ Humanigen } & \multirow[b]{2}{*}{ GM-CSF } & Phase 2 & NCT04583969 (Recruiting) & 2021 \\
\hline & & & & Phase 3 & $\begin{array}{c}\text { NCT04351152 (Recruiting), } \\
\text { NCT04534725 (Not yet recruiting) }\end{array}$ & 2020 \\
\hline & Gimsilumab & $\begin{array}{l}\text { Kinevant Sciences } \\
\text { Roivant Sciences }\end{array}$ & GM-CSF & Phase 2 & NCT04351243 (Active, not recruiting) & $2020 / 2021$ \\
\hline & TJ003234 & I-Mab Biopharma & GM-CSF & Phase $2 / 3$ & NCT04341116 (Recruiting) & 2021 \\
\hline & \multirow[t]{2}{*}{ Mavrilimumab } & \multirow[t]{2}{*}{$\begin{array}{c}\text { Kiniksa } \\
\text { pharmaceuticals }\end{array}$} & \multirow[t]{2}{*}{ GM-CSF-R } & Phase 2 & $\begin{array}{l}\text { NCT04397497 (Not yet recruiting), } \\
\text { NCT04463004 (Recruiting), } \\
\text { NCT04399980 (Active, not recruiting), } \\
\text { NCT04492514 (Recruiting) }\end{array}$ & $2020-2021$ \\
\hline & & & & Phase $2 / 3$ & NCT04447469 (Recruiting) & 2021 \\
\hline & Pamrevlumab & FibroGen & CTGF & Phase 2 & NCT04432298 (Recruiting) & $2020 / 2021$ \\
\hline & $\begin{array}{l}\text { SNDX-6352 } \\
\text { (axatilimab) }\end{array}$ & $\begin{array}{c}\text { Syndax } \\
\text { Pharmaceuticals }\end{array}$ & CSF-1R & Phase 2 & NCT04415073 (Suspended) & 2020 \\
\hline & & & & Phase 2 & $\begin{array}{l}\text { NCT04344782 (Active, Not yet } \\
\text { recruiting), NCT04275414 (Completed) }\end{array}$ & 2020 \\
\hline & BDB-001 & $\begin{array}{l}\text { Staidson } \\
\text { Biopharmaceuticals }\end{array}$ & PD-L1 & Phase $2 / 3$ & NCT04449588 (Recruiting) & $2021 / 2022$ \\
\hline & \multirow{4}{*}{ Nivolumab } & \multirow{4}{*}{$\begin{array}{l}\text { Bristol-Myers } \\
\text { Squibb }\end{array}$} & \multirow{4}{*}{ PD1 } & Phase 2 & $\begin{array}{l}\text { NCT04413838 (Active, Not } \\
\text { yet recruiting) }\end{array}$ & 2021 \\
\hline & & & & Phase 2 & $\begin{array}{l}\text { NCT04343144 (Active, Not } \\
\text { yet recruiting) }\end{array}$ & 2020 \\
\hline & & & & Phase 2 & NCT04356508 (Not yet recruiting) & 2021 \\
\hline & & & & Phase 2 & NCT04356508S & $2020 / 2021$ \\
\hline & Crizanlizumab & Novartis & P-selectin & Phase 2 & $\begin{array}{l}\text { NCT04435184 (Recruiting) } \\
\text { NCT03474965 (Recruiting) }\end{array}$ & $\begin{array}{l}2020 \\
2024\end{array}$ \\
\hline & $\begin{array}{c}\text { CSL312 } \\
\text { (garadacimab) }\end{array}$ & CSL Behring & Factor XIIa & Phase 2 & NCT04409509 (Recruiting) & 2020 \\
\hline & LY3127804 & Eli Lilly & Angiopoietin 2 & Phase 2 & NCT04342897 (Terminated) & 2020 \\
\hline & \multirow[t]{2}{*}{ Ravulizumab } & \multirow{2}{*}{$\begin{array}{c}\text { Alexion } \\
\text { pharmaceuticals }\end{array}$} & \multirow{2}{*}{ C5 } & Phase 3 & $\begin{array}{l}\text { NCT04369469 (Recruiting) } \\
\text { NCT04570397 (Not yet recruiting) }\end{array}$ & $2020 / 2021$ \\
\hline & & & & Phase 4 & NCT04390464 (Recruiting) & $2021 / 2022$ \\
\hline & Secukinumab & Novartis & IL-17A & Phase 2 & NCT04403243 (Recruiting) & 2020 \\
\hline & \multirow{2}{*}{ Canakinumab } & \multirow{2}{*}{ Novartis } & \multirow{2}{*}{ IL-1ß } & Phase 2 & $\begin{array}{l}\text { NCT04365153 (Active, Not } \\
\text { yet recruiting) }\end{array}$ & 2020 \\
\hline & & & & Phase 3 & $\begin{array}{l}\text { NCT04362813 (Active, not recruiting) } \\
\text { NCT04510493 (Recruiting) }\end{array}$ & $\begin{array}{l}2020 \\
2023\end{array}$ \\
\hline
\end{tabular}


Table 3. Cont.

\begin{tabular}{|c|c|c|c|c|c|c|}
\hline Strategy & Generic Name & $\begin{array}{l}\text { Sponsoring } \\
\text { Company }\end{array}$ & Target & $\begin{array}{l}\text { Development } \\
\text { Stage }\end{array}$ & NCT (Status) & $\begin{array}{l}\text { Completion } \\
\text { Date }\end{array}$ \\
\hline & \multirow[t]{2}{*}{ Clazakizumab } & \multirow[t]{2}{*}{ Vitaeris } & \multirow[t]{2}{*}{ IL-6 } & \multirow[t]{2}{*}{ Phase 2} & $\begin{array}{c}\text { NCT04381052 (Not yet recruiting), } \\
\text { NCT04343989 (Recruiting), } \\
\text { NCT04363502 (Recruiting), } \\
\text { NCT04494724 (Recruiting), } \\
\text { NCT04348500 (Not yet recruiting) } \\
\text { NCT04659772 (Enrolling by invitation) }\end{array}$ & $2020 / 2021$ \\
\hline & & & & & NCT04348500 (Active, not recruiting) & 2021 \\
\hline & Sirukumab & Janssen & IL-6 & Phase 2 & NCT04380961 (Recruiting) & 2021 \\
\hline & \multirow{4}{*}{ Sarilumab } & \multirow{4}{*}{$\begin{array}{c}\text { Regeneron } \\
\text { Pharmaceuticals }\end{array}$} & \multirow{4}{*}{ IL-6 } & Phase 1 & $\begin{array}{l}\text { NCT04386239 (Active, Not } \\
\text { yet recruiting) }\end{array}$ & 2020 \\
\hline & & & & Phase 2 & $\begin{array}{c}\text { NCT04357860 (Not yet recruiting), } \\
\text { NCT04357808 (Active, not recruiting), } \\
\text { NCT04359901 (Recruiting), } \\
\text { NCT04661527 (Recruiting) }\end{array}$ & $2020 / 2023$ \\
\hline & & & & Phase $2 / 3$ & $\begin{array}{c}\text { NCT04315298 (Completed), } \\
\text { NCT04324073 (Active, not recruiting), } \\
\text { NCT04341870 (Suspended) }\end{array}$ & 2021 \\
\hline & & & & Phase 3 & $\begin{array}{l}\text { NCT04327388 (Completed), } \\
\text { NCT04345289 (Recruiting) }\end{array}$ & $2020 / 2021$ \\
\hline & Olokizumab & $\begin{array}{l}\text { R-Pharm } \\
\text { International }\end{array}$ & IL-6 & Phase $2 / 3$ & $\begin{array}{c}\text { NCT04380519 (Completed), } \\
\text { NCT04452474 (Not yet recruiting) }\end{array}$ & 2020-2021 \\
\hline & $\begin{array}{l}\text { Tozilizumab + } \\
\text { Sarilumab }\end{array}$ & Roche, Sanofi & IL-6 & Phase 2 & NCT04322773 (Terminated) & 2021 \\
\hline & Siltuximab & Janssen & IL-6 & Phase 2 & NCT04329650 (Recruiting) & 2020 \\
\hline \multirow{7}{*}{$\begin{array}{l}\text { ARDS- } \\
\text { related } \\
\text { target }\end{array}$} & \multirow{5}{*}{ Tocilizumab } & \multirow{5}{*}{ Roche } & \multirow{5}{*}{ IL-6R } & Phase 1 & NCT04560205 (Recruiting) & 2020 \\
\hline & & & & Phase 2 & $\begin{array}{c}\text { NCT04445272 (Recruiting), } \\
\text { NCT04479358 (Recruiting), } \\
\text { NCT04412291 (Recruiting), } \\
\text { NCT04331795 (Completed), } \\
\text { NCT04377659 (Active, not recruiting), } \\
\text { NCT04363736 (Completed), } \\
\text { NCT04435717 (Recruiting), } \\
\text { NCT04377503 (Active, Not yet } \\
\text { recruiting), NCT04339712 (Recruiting), } \\
\text { NCT04370834 (Suspended), } \\
\text { NCT04315480 (Active, not recruiting), } \\
\text { NCT04331808 (Active, not recruiting), } \\
\text { NCT04317092 (Active, Not yet } \\
\text { recruiting) NCT04363853 (Active, Not } \\
\text { yet recruiting) }\end{array}$ & 2020-2022 \\
\hline & & & & Phase $2 / 3$ & NCT04381936 & $2021 / 2031$ \\
\hline & & & & Phase 3 & $\begin{array}{c}\text { NCT04403685 (Terminated), } \\
\text { NCT04423042 (Not yet recruiting), } \\
\text { NCT04412772 (Recruiting), } \\
\text { NCT04345445 (Not yet recruiting), } \\
\text { NCT04424056 (Not yet recruiting), } \\
\text { NCT04335071 (Terminated), } \\
\text { NCT04361032 (Not yet recruiting), } \\
\text { NCT04320615 (Completed), } \\
\text { NCT04372186 (Active, not recruiting), } \\
\text { NCT04356937 (Active, not recruiting), } \\
\text { NCT04577534 (Recruiting) }\end{array}$ & 2020-2022 \\
\hline & & & & Phase 4 & NCT04377750 (Recruiting) & $2020 / 2021$ \\
\hline & Levilimab & Biocad & IL-6R & Phase 3 & NCT04397562 (Completed) & 2021 \\
\hline & $\begin{array}{l}\text { Tocilizumab + } \\
\text { Dexametha- } \\
\text { sone }\end{array}$ & Roche & IL-6R & Phase 2 & NCT04476979 (Recruiting) & 2021 \\
\hline
\end{tabular}


Table 3. Cont.

\begin{tabular}{|c|c|c|c|c|c|c|}
\hline Strategy & Generic Name & $\begin{array}{l}\text { Sponsoring } \\
\text { Company }\end{array}$ & Target & $\begin{array}{l}\text { Development } \\
\text { Stage }\end{array}$ & NCT (Status) & $\begin{array}{l}\text { Completion } \\
\text { Date }\end{array}$ \\
\hline \multirow{22}{*}{$\begin{array}{l}\text { ARDS- } \\
\text { related } \\
\text { target }\end{array}$} & $\begin{array}{c}\text { Tocilizumab + } \\
\text { Remdesivir }\end{array}$ & Roche & IL-6R & Phase 3 & NCT04409262 (Recruiting) & 2020 \\
\hline & $\begin{array}{c}\text { Tocilizumab + } \\
\text { Favipiravir }\end{array}$ & Roche & IL-6R & NA & NCT04310228 (Recruiting) & 2020 \\
\hline & $\begin{array}{l}\text { Tocilizumab } \\
\text { +Siltuximab }\end{array}$ & Roche, Janssen & IL-6 + IL-6R & Phase 3 & NCT04330638 (Active, not recruiting) & 2020 \\
\hline & $\begin{array}{c}\text { Tocilizumab + } \\
\text { Pem- } \\
\text { brolizumab }\end{array}$ & Roche, Merck & IL-6R + PD-1 & Phase 2 & NCT04335305 (Recruiting) & 2020 \\
\hline & $\begin{array}{c}\text { Tocilizumab + } \\
\text { Hydroxychloro- } \\
\text { quine }+ \\
\text { Azithromycin }\end{array}$ & Roche & IL-6R & Phase 2 & NCT04332094 (Recruiting) & 2020 \\
\hline & $\begin{array}{l}\text { Heparin }+ \\
\text { Tocilizumab }\end{array}$ & Roche & IL-6R & Phase 3 & NCT04600141 (Not yet recruiting) & 2021 \\
\hline & BMS-986253 & $\begin{array}{l}\text { Bristol-Myers } \\
\text { Squibb }\end{array}$ & IL-8 & Phase 2 & NCT04347226 (Recruiting) & $2021 / 2022$ \\
\hline & \multirow{2}{*}{ Risankizumab } & \multirow{2}{*}{$\begin{array}{l}\text { Jiangsu Pacific } \\
\text { Meinuoke Bio } \\
\text { Pharmaceutical }\end{array}$} & \multirow{2}{*}{ IL-23 } & Phase 2 & NCT04583956 (Recruiting) & 2021 \\
\hline & & & & Phase $2 / 3$ & NCT04586153 (Not yet recruiting) & 2021 \\
\hline & $\begin{array}{l}\text { MSTT1041A } \\
\text { (astegolimab) }\end{array}$ & Genetech & IL-33 & Phase 2 & NCT04386616 (Recruiting) & 2020 \\
\hline & Emapalumab & $\begin{array}{l}\text { Swedish Orphan } \\
\text { BIovitrum }\end{array}$ & IFN- $\gamma$ & Phase $2 / 3$ & NCT04324021 (Terminated) & 2020 \\
\hline & $\begin{array}{c}\text { IFX1 } \\
\text { (vilobelimab) }\end{array}$ & InflaRx GmbH & C5a & Phase2/3 & NCT04333420 (Recruiting) & 2021 \\
\hline & Infliximab & MSD & TNF-alpha & Phase 2 & NCT04425538 (Recruiting) & 2020 \\
\hline & $\begin{array}{l}\text { Remdesivir }+ \\
\text { infliximab }\end{array}$ & MSD & TNF-alpha & Phase 3 & NCT04593940 (Recruiting) & 2021 \\
\hline & Eculizumab & $\begin{array}{c}\text { Alexion } \\
\text { pharmaceuticals }\end{array}$ & C5 & Phase 2 & NCT04346797 (Recruiting) & 2020 \\
\hline & AK119 & Akesobio & CD73 & Phase 1 & NCT04516564 (Recruiting) & 2021 \\
\hline & CERC-002 & Cerecor & LIGHT & Phase 2 & NCT04412057 (Recruiting) & 2020 \\
\hline & Glenzocimab & Acticor Biotech & $\begin{array}{c}\text { Platelet } \\
\text { glycoprotein VI }\end{array}$ & Phase 2 & NCT04659109 (Not yet recruiting) & 2021 \\
\hline & VIB7734 & Viela Bio & ILT7 & Phase 1 & NCT04526912 (Recruiting) & 2021 \\
\hline & NGM621 & ngmbio & $\mathrm{C} 3$ & Phase $1 / 2$ & NCT04582318 (Recruiting) & 2021 \\
\hline & EB05 & Edesa Biotech & TLR4 & Phase $2 / 3$ & NCT04401475 (Not yet recruiting) & 2021 \\
\hline & Avdoralimab & Innate Pharma & $\mathrm{C} 5 \mathrm{aR}$ & Phase 2 & NCT04371367 (Recruiting) & 2020 \\
\hline \multirow{8}{*}{$\begin{array}{l}\text { Virus- } \\
\text { related } \\
\text { target }\end{array}$} & BRII-196 & \multirow{2}{*}{ Brii biosciences } & $\mathrm{S}$ protein & Phase 1 & NCT04479631 (Active, not recruiting) & 2021 \\
\hline & BRII-198 & & $\mathrm{S}$ protein & Phase 1 & NCT04479644 (Active, not recruiting) & 2021 \\
\hline & JS016 & $\begin{array}{l}\text { Shanghai Junshi } \\
\text { Bioscience } \\
\text { Co., Ltd. }\end{array}$ & $S$ protein & Phase 1 & NCT04441918 (Recruiting) & 2020 \\
\hline & \multirow{5}{*}{$\begin{array}{l}\text { LY-CoV555, } \\
\text { LY3819253 }\end{array}$} & \multirow{5}{*}{ Eli Lilly } & \multirow{5}{*}{$\mathrm{S}$ protein } & Phase 1 & $\begin{array}{c}\text { NCT04411628 (Completed), } \\
\text { NCT04537910 (Active, not recruiting) }\end{array}$ & 2020 \\
\hline & & & & Phase 2 & NCT04427501 (Recruiting) & 2020 \\
\hline & & & & Phase $2 / 3$ & NCT04518410 (Recruiting) & 2021 \\
\hline & & & & Phase 3 & $\begin{array}{l}\text { NCT04497987 (Recruiting), } \\
\text { NCT04501978 (Active, not recruiting) }\end{array}$ & 2021 \\
\hline & & & & Phase 4 & NCT04656691 (Not yet recruiting) & 2021 \\
\hline
\end{tabular}


Table 3. Cont.

\begin{tabular}{|c|c|c|c|c|c|c|}
\hline Strategy & Generic Name & $\begin{array}{l}\text { Sponsoring } \\
\text { Company }\end{array}$ & Target & $\begin{array}{l}\text { Development } \\
\text { Stage }\end{array}$ & NCT (Status) & $\begin{array}{l}\text { Completion } \\
\text { Date }\end{array}$ \\
\hline \multirow{26}{*}{$\begin{array}{l}\text { Virus- } \\
\text { related } \\
\text { target }\end{array}$} & \multirow{4}{*}{$\begin{array}{c}\text { REGN-COV2 } \\
\text { (REGN10933 + } \\
\text { REGN10987) }\end{array}$} & \multirow{4}{*}{$\begin{array}{c}\text { Regeneron } \\
\text { Pharmaceuticals }\end{array}$} & \multirow{4}{*}{$S$ protein } & Phase 1 & NCT04519437 (Active, not recruiting) & 2021 \\
\hline & & & & Phase $1 / 2$ & $\begin{array}{l}\text { NCT04425629 (Recruiting), } \\
\text { NCT04426695 (Recruiting) }\end{array}$ & 2020-2021 \\
\hline & & & & Phase 2 & NCT04666441 (Not yet recruiting) & 2021 \\
\hline & & & & Phase 3 & NCT04452318 (Recruiting) & 2021 \\
\hline & \multirow{2}{*}{ SCTA01 } & \multirow{2}{*}{ Sinocelltech } & \multirow{2}{*}{$\mathrm{S}$ protein } & Phase 1 & NCT04483375 (Recruiting) & 2021 \\
\hline & & & & Phase $2 / 3$ & NCT04644185 (Not yet recruiting) & 2021 \\
\hline & $\begin{array}{l}\text { STI-2020 (COVI- } \\
\left.\text { AMG }^{\mathrm{TM}}\right)\end{array}$ & $\begin{array}{l}\text { Sorrento } \\
\text { Therapeutics }\end{array}$ & $\mathrm{S}$ protein & Phase $1 / 2$ & NCT04584697 (Recruiting) & 2021 \\
\hline & $\begin{array}{l}\text { COVI-GUARD } \\
\text { (STI-1499) }\end{array}$ & $\begin{array}{l}\text { Sorrento } \\
\text { Therapeutics }\end{array}$ & $\mathrm{S}$ protein & Phase 1 & NCT04454398 (Recruiting) & 2021 \\
\hline & \multirow{2}{*}{ TY027 } & \multirow{2}{*}{ Tychan Pte Ltd. } & \multirow{2}{*}{$\mathrm{S}$ protein } & Phase 1 & NCT04429529 (Active, not recruiting) & 2020 \\
\hline & & & & Phase 3 & NCT04649515 (Not yet recruiting) & 2021 \\
\hline & \multirow{2}{*}{ BGB-DXP593 } & \multirow{2}{*}{ BeiGene } & \multirow{2}{*}{$\mathrm{S}$ protein } & Phase 1 & NCT04532294 (Recruiting) & 2021 \\
\hline & & & & Phase 2 & NCT04551898 (Not yet recruiting) & 2021 \\
\hline & VIR-7831 & $\begin{array}{c}\text { Vir } \\
\text { Biotechnology- } \\
\text { GlaxoSmithKline }\end{array}$ & $S$ protein & Phase 2/3 & NCT04545060 (Recruiting) & 2021 \\
\hline & \multirow{2}{*}{ MW33 } & \multirow{2}{*}{$\begin{array}{l}\text { Mabwell } \\
\text { Bioscience }\end{array}$} & \multirow{2}{*}{$\mathrm{S}$ protein } & Phase 1 & NCT04533048 (Active, not recruiting) & 2020 \\
\hline & & & & Phase 2 & NCT04627584 (Not yet recruiting) & 2021 \\
\hline & 47D11 & AbbVie & $S$ protein & Phase 1 & NCT04644120 (Not yet recruiting) & 2021 \\
\hline & \multirow{2}{*}{$\begin{array}{c}\text { AZD7442 } \\
(\mathrm{AZD} 8895+ \\
\text { AZD1061) }\end{array}$} & \multirow[b]{2}{*}{ AstraZeneca } & \multirow[b]{2}{*}{$\mathrm{S}$ protein } & Phase 1 & NCT04507256 (Active, not recruiting) & 2021 \\
\hline & & & & Phase 3 & $\begin{array}{l}\text { NCT04625725 (Recruiting), } \\
\text { NCT04625972 (Recruiting) }\end{array}$ & 2022 \\
\hline & LY3832479 & Junshi/Eli Lilly & & Phase $2 / 3$ & NCT04441931 (Completed) & 2020 \\
\hline & HFB30132A & Hifibio & $\mathrm{S}$ protein & Phase1 & NCT04590430 (Recruiting) & 2021 \\
\hline & $\begin{array}{l}\text { BI767551 } \\
\text { DZIF-10c }\end{array}$ & $\begin{array}{l}\text { Boehringer } \\
\text { Ingelheim }\end{array}$ & & Phase 1/2 & $\begin{array}{l}\text { NCT04631705 (Not yet recruiting) } \\
\text { NCT04631666 (Not yet recruiting) }\end{array}$ & 2021 \\
\hline & $\begin{array}{l}\text { HLX71 fusion } \\
\text { protein }\end{array}$ & $\begin{array}{l}\text { Hengenix } \\
\text { biotech/Henlius }\end{array}$ & $\mathrm{S}$ protein & Phase1 & NCT04583228 (Not yet recruiting) & 2021 \\
\hline & HLX70 & $\begin{array}{l}\text { Hengenix } \\
\text { biotech/Henlius }\end{array}$ & $S$ protein & Phase $1 / 2$ & NCT04561076 (Not yet recruiting) & \\
\hline & AMD03820 & Olygo Bioservice & & Phase $1 / 2$ & NCT04592549 (Recruiting) & 2021 \\
\hline & \multirow{2}{*}{ CT-P59 } & \multirow{2}{*}{ Celltrion } & \multirow{2}{*}{$\mathrm{S}$ protein } & Phase $1 / 2$ & NCT04525079 (Recruiting) & 2020 \\
\hline & & & & Phase $2 / 3$ & NCT04602000 (Recruiting) & 2021 \\
\hline
\end{tabular}

CCR, CC chemokine receptor; CD, cluster of differentiation; CSF1R, colony stimulating factor 1 receptor; CTGF, connective-tissue growth factor; GM-CSF, granulocyte monocyte colony stimulating factor; GM-CSF-R, granulocyte monocyte colony stimulating factor receptor; IFN- $\gamma$, interferon gamma; IL, interleukin; IL-R, interleukin receptor; PD1, programmed cell death 1; S protein, spike protein; TNF, tumor necrosis factor; VEGF, vascular endothelial growth factor; VEGFR, vascular endothelial growth factor receptor.

Regarding the first category, several clinical trials are evaluating the potential use of antibodies targeting pro-inflammatory cytokines or their receptors. Among them, two are already approved for the treatment of COVID-19 in Russia: olokizumab and levilimab. Olokizumab, a humanized anti-IL6 IgG4 (R-Pharm International), was approved in May 2020, for patients suffering from severe COVID-19 respiratory distress. Levilimab (Biocad) is a human IgG1 that binds to soluble and membrane-bound IL-6 receptor and was also approved in Russia, in June 2020. In addition, re-purposed Abs, already approved as treatments for other diseases, are the subject of strong interest with numerous clinical trials. Sarilumab (Sanofi/Regeneron) is a human IgG1 targeting the interleukin-6 receptor. It is approved for rheumatoid arthritis in adults and currently tested in Phase 3 for 
hospitalized patients with COVID-19, alone or in association to tocilizumab. Tocilizumab (Hoffmann-LaRoche) is a humanized IgG1 also targeting IL-6R, marketed for several indications and is currently tested in 42 clinical trials in COVID-19 patients. First results in Europe are promising, showing an improvement of clinical outcome in patients with moderate or severe COVID-19 pneumonia [46]. Siltuximab (EUSA Pharma, Hempstead, UK and BeiGene Ltd., Beijing, China) is a chimeric IgG1 targeting IL-6, indicated in patients with multicentric Castleman's disease and evaluated in Phase 3 in COVID-19 (NCT04330638). Siltuximab already showed promising results in patients with COVID-19 respiratory failure, when used as compassionate treatment [47]. Emapalumab (Swedish Orphan Biovitrum) is a human IgG1 raised against interferon gamma, approved for treatment of primary hemophagocytic lymphohistiocytosis, and evaluated in a Phase $2 / 3$ in patients with COVID-19 (NCT04324021). Considering the involvement of the dysregulated complement cascade in severe COVID-19 complications, ravulizumab (Alexion Pharmaceuticals), a humanized IgG2/4 directed against C5, marketed for treatment of paroxysmal nocturnal hemoglobinuria, was evaluated in Phase 3 (NCT04369469).

The second category of anti-COVID-19 Abs, corresponding to more than half (including discovery and preclinical stages), directly targets SARS-CoV2. The majority are raised against $S$ proteins to prevent binding of the virus on host cells. Among the Ab-based therapeutics in development, there are polyclonal Abs, fusion proteins, single domain Abs and DARPin, but the majority are monoclonal Abs. Among them, eight molecules already reached clinical trials: some are proposed as an antibody cocktail (REGN-COV2, AZD7442, AMD03820), some were purified from a convalescent patient (LY3819253, CT-P59, JS016, AZD7442, HFB30132A) and most of them are fully-human monoclonal Abs. Several of those Abs have already reached phase 3 (LY-CoV555/LY3819253, REGN-COV2, SCTA01, TY027, VIR-7831, AZD7442, LY3832479, CT-P59) and two of them (LY3819253, REGNCOV2) received an Emergency Use Authorization (EUA) [48]. However, the Abs most likely to be authorized in the next months are Abs from plasma of recovering COVID-19 patients, which may benefit to hospitalized COVID-19 patients until other biotherapeutics are available. It is noteworthy that an antibody is evaluated by the inhalation route as well as by intravenous injection (BI767551 DZIF-10c, Boehringer Ingelheim). A matter of concern for the development of vaccines or therapeutic Abs against SARS-CoV2 is antibody-dependent enhancement of viral entry (ADE), enhancing viral entry into cells expressing the Fc receptor for immunoglobulins. Although ADE has not been demonstrated yet for SARS-CoV-2, neutralizing Abs without an effector function may be appropriate to prevent ADE.

\subsection{Therapeutic Abs against Bacterial Diseases}

With the exception of Haemophilus influenza type B mediating severe epiglottitis and laryngotracheitis and Streptococcus pyogenes causing pharyngitis, and for which no antibodies are under development, bacteria are mostly associated with lower respiratory tract infections (mainly pneumonia) [49]. The most common etiologic agent of pneumonia is Streptococcus pneumoniae, which has a greater incidence in children and the elderly [50]. Other frequent causes of bacterial pneumonia, often with a multidrug-resistant phenotype, include Pseudomonas aeruginosa, Staphylococcus aureus, Haemophilus influenza and also bacteria grouped as atypical pneumonia (Mycoplasma pneumoniae, Chlamydia spp., Legionella spp. or Coxiella burnetii) [50].

Pneumonia is characterized by an infection of the lung parenchyma. It occurs when the immune system is unable to eliminate a pathogen from the alveolar compartment [51]. The impairment of host immunity is either due to a pathogen's active mechanisms (toxin or virulence factors production) or to comorbidities (immunodeficiency, impaired mucociliary clearance, airway obstruction). The pathogen gives rise to a protracted local inflammation, which will alter lung mechanics and physiology and perpetuate damage to the lung parenchyma, accelerating systemic inflammation and worsening prognosis [52]. In the next 
sections, we will present and discuss the most recent advances in Abs targeting bacterial pathogens involved in RTI (Table 4).

Table 4. Therapeutic antibodies for bacterial respiratory infections.

\begin{tabular}{|c|c|c|c|c|c|c|c|}
\hline Indication & Generic Name & $\begin{array}{l}\text { Sponsoring } \\
\text { Company }\end{array}$ & Target & $\begin{array}{l}\text { Development } \\
\text { Stage }\end{array}$ & NCT & $\begin{array}{c}\text { Completion } \\
\text { Date }\end{array}$ & Status \\
\hline \multirow{2}{*}{$\begin{array}{c}\text { Staphylococcus } \\
\text { aureus }\end{array}$} & MEDI4893 & AstraZeneca & $\alpha$-toxin & Phase 2 & NCT02296320 & 2018 & \multirow{2}{*}{$\begin{array}{c}\text { Active on AZ } \\
\text { pipeline } \\
\text { Active on } \\
\text { Aridis pipeline }\end{array}$} \\
\hline & AR-301 & $\begin{array}{c}\text { Aridis } \\
\text { Pharmaceuticals }\end{array}$ & $\alpha$-toxin & Phase 3 & NCT03816956 & 2020 & \\
\hline $\begin{array}{l}\text { Bacillus } \\
\text { anthracis }\end{array}$ & $\begin{array}{l}\text { Obiltoxaximab } \\
\text { (anthim) }\end{array}$ & $\begin{array}{c}\text { Elusys } \\
\text { Therapeutics }\end{array}$ & PA & Phase 4 & NCT03088111 & 2020 & \\
\hline \multirow{2}{*}{$\begin{array}{l}\text { Pseudomonas } \\
\text { aeruginosa }\end{array}$} & $\begin{array}{c}\text { AR-101 } \\
\text { (aerumab) }\end{array}$ & $\begin{array}{c}\text { Aridis } \\
\text { Pharmaceuticals }\end{array}$ & LPS & Phase 2 & NCT00851435 & 2009 & \multirow{2}{*}{$\begin{array}{c}\text { Active on } \\
\text { Aridis pipeline } \\
\text { Active on } \\
\text { Aridis pipeline }\end{array}$} \\
\hline & $\begin{array}{l}\text { AR-105 } \\
\text { (aerucin) }\end{array}$ & $\begin{array}{c}\text { Aridis } \\
\text { Pharmaceuticals }\end{array}$ & Alginate & Phase2 & NCT03027609 & 2019 & \\
\hline
\end{tabular}

LPS, lipopolysaccharide; PA, protective antigen.

\subsubsection{Bacillus anthracis}

Bacillus anthracis is a spore-forming, rod-shaped, Gram-positive, facultative anaerobic bacterium, responsible for a zoonotic disease called anthrax. Humans can develop anthrax through exposure to infected animals or their products. The clinical manifestation of human anthrax varies accordingly to the mode of entry of the bacterium, with pulmonary anthrax (inhalation of spores) being the most fatal form. Apart from its animal origin, anthrax is considered to be a major bioterrorism threat. After inhalation, clinical symptoms develop usually within a week and resemble those of influenza or community-acquired pneumonia [53]. Without any treatment, pulmonary anthrax mortality ranges between 50 and $80 \%$ and coincides with the production of two main virulence factors: A capsule, which precludes the phagocytosis by host immune cells, and the tripartite anthrax toxin. Anthrax toxin is composed of a protective antigen (PA), the edema factor (EF) and the lethal factor (LF) [54]. PA allows the binding, molecular re-arrangement and endocytosis of EF and LF in host cells, ultimately causing swelling and cell death. The essential role of PA in the pathophysiology of anthrax has been extensively documented and makes this protein an attractive therapeutic target [55]. Bioterrorism anthrax attacks in the USA in 2001 provoked massive investments in medical countermeasures, which resulted in the marketing of raxibacumab (ABthrax ${ }^{\circledR}$-2012) and obiltoxaximab (Anthim ${ }^{\circledR}$-2016), two Abs that neutralize PA [56,57]. Raxibacumab is a human $\operatorname{IgG}_{1}$, whereas obiltoxaximab is a chimeric IgG Ig $_{1}$ A Phase 3 study in 333 human volunteers (NCT00639678) showed higher tissue concentrations of raxibacumab than those that were shown effective in animals [57]. In monkeys, obiltoxaximab allowed $100 \%$ survival if administered one to three days before anthrax exposure, and 83 to $100 \%$ survival, if given 18 to $24 \mathrm{~h}$ after challenge [57]. The two Abs are indicated in adult and pediatric patients for the treatment of pulmonary anthrax in combination with standard-of-care antibacterial drugs. It is noteworthy that there is not any data related to pediatric populations, and the dose level and regimen were extrapolated from a population-based PK approach. In the clinical practice, raxibacumab is indicated for the treatment of anthrax pulmonary disease in adults at the intravenous dose of $40 \mathrm{mg} / \mathrm{kg}$, in children at 40 to $80 \mathrm{mg} / \mathrm{kg}$ in combination with adequate conventional antibiotics, and in prophylaxis if alternatives are not feasible. Obiltoxaximab is used in the same indications at the dosages of $16 \mathrm{mg} / \mathrm{kg}$ in adults and 16 to $32 \mathrm{mg} / \mathrm{kg}$ in children. However, premedication with diphenhydramine is recommended prior to obiltoxaximab administration, because it was reported to induce hypersensitivity reactions and anaphylaxis in healthy subjects [57], when it was given intravenously at $16 \mathrm{mg} / \mathrm{kg}$. Due to the concern about adverse reactions, a Phase 4 trial has recently started (NCT03088111) to assess the clinical benefit of the use of obiltoxaximab given intravenously. Recently, very good tolerance of obiltoxaximab in a 
randomized open-label Phase 1 trial in healthy volunteers (NCT01952444) by intramuscular route (up to $24 \mathrm{mg} / \mathrm{kg}$ ) was reported [58].

\subsubsection{Staphylococcus aureus}

Staphylococcus aureus is an opportunistic Gram-positive pathogen with a significant worldwide healthcare burden. A substantial part of the human population is naturally colonized by this bacterium. Nonetheless, S. aureus has the ability to change from a commensal phenotype to an actual pathogen involved in various clinical manifestations, of which the most serious is pneumonia, especially in hospitalized patients [59]. The recent increasing prevalence of methicillin-resistant Staphylococcus aureus (MRSA) strains has become a global health problem attributable to a high mortality rate [60], complicating antimicrobial therapy. In contrast to most bacterial pathogens, S. aureus has the ability to secrete a large array of cytotoxins [61]. In particular, alpha-hemolysin (Hla or AT) is a key virulence factor. Once bound to its receptor (ADAM10) on the host cell surface, Hla oligomerizes to form heptameric transmembrane pores, which may lyse epithelial, endothelial and immune cells. To overcome host defenses, S. aureus also produces five leukocidins (SF-PV, LukED, LukGH, HlgAB and HlgCB), which target myeloid and lymphoid cells [62].

Two Abs are currently under investigation and aim at preventing or limiting S. aureus-related disease through the neutralization of AT. MEDI-4893 (suvratoxumab, by AstraZeneca) has been shown to be protective in multiple animal models of pneumonia [63-65]. It is a high affinity IgG1 with an extended T1/2 (YTE mutated). During a Phase 2 trial, it was shown to slightly improve lung function of patients with S. aureus infection and was associated with low adverse events, overall encouraging AstraZeneca to continue its clinical development [66]. AR-301 (salvecin), developed by Aridis Pharmaceuticals, was designed as an adjunctive therapy to standard-of-care anti-S. aureus antibiotics. Even if there was no preclinical data published, the results of a Phase 2 trial were recently presented and showed a significant reduction in time under mechanical ventilation in $\mathrm{Ab}$ - plus antibiotics-treated patients as compared to the placebo, with good tolerance [67]. These promising results prompted a Fast Track designation by the FDA (Food and Drug Agency), an orphan-drug designation by the EMA (European Medicines Agency) and the design of a Phase 3 trial (NCT03816956), which is currently enrolling patients.

\subsubsection{Pseudomonas aeruginosa}

Pseudomonas aeruginosa $(\mathrm{Pa})$ is another important nosocomial pathogen responsible for devastating acute pneumonia, in immunocompromised, ventilated patients or cystic fibrosis patients with immunologically-quiet chronic colonization. Pa versatility is related to the genetic flexibility of its large genome [68]. Many bacterial surface antigens have been considered for the development of therapeutic Abs, with disappointing results. For instance, the most clinically advanced Ab is AR-101 (aerumab), a human IgM binding to lipopolysaccharide (LPS) serotype O11. LPS is an important component of the P. aeruginosa outer membrane and has been historically used for serologic typing before genomic methods were available. In a Phase 2 trial, AR-101 given in combination with standardof-care anti- $P$. aeruginosa antibiotics proved to be well tolerated and decrease the time to pneumonia resolution as compared to the placebo [69]. Additional bacterial antigens have been considered for the development of Abs in P. aeruginosa strains associated with chronic colonization. Aridis Pharmaceuticals developed AR-105 (aerucin), a human IgG1, directed against $P$. aeruginosa biofilm - a complex extracellular polymeric matrix that is produced by the bacteria to increase its resistance to the host's immune cells and against antimicrobial therapy, thereby favoring its long-term survival in the host. AR-105 targets alginate, one of the three main exopolysaccharides (with PsI, Pel) composing the biofilm [70]. It successfully completed a Phase 1 clinical trial in 16 healthy volunteers, with a good tolerance up to $20 \mathrm{mg} / \mathrm{kg}$. The FDA has granted AR-105 with a fast-track designation and Aridis Pharmaceuticals initiated a Phase 2 trial in the second quarter of 2017. Results were expected to be reported in the third quarter of 2019, but there is not any information yet. 


\subsection{Therapeutic Abs against Fungal Diseases}

Few therapeutic $\mathrm{Ab}$ have been developed against fungal diseases. Allergic bronchopulmonary aspergillosis (ABPA) is the main focus of current research interests (Table 5). ABPA usually develops in patients with chronic respiratory diseases, particularly in asthmatics or in pediatric cystic fibrosis patients. Triggered by hypersensitivity immune reactions to Aspergillus antigens in a context of chronic Aspergillus tracheobronchial colonization, ABPA has an inflammatory pathology, characterized in particular by augmented titers in specific anti-Aspergillus IgE [71]. The main objective of ABPA therapy is to control episodes of acute inflammation and limit the progression of lung injury. Two Abs, benralizumab and dupilumab, are under clinical trials and target components of the host immune responses. Benralizumab is a humanized IgG1 that binds to the IL-5R $\alpha$ subunit, on eosinophils and basophils. The absence of fucose on the Fc fragment of benralizumab results in a high affinity for Fc $\gamma$ RIII receptors present on the surface of immune effector cells, such as natural killer cells. This causes apoptosis of eosinophils and basophils through ADCC. In 2019, a Japanese team reported the case of a patient with ABPA successfully treated with benralizumab [72]. A Phase 4 (NCT04108962) open-label study is now evaluating the effects of benralizumab in the treatment of severe asthma in patients with allergic bronchopulmonary aspergillosis. Dupilumab is a humanized IgG4 that binds to the IL-4R $\alpha$ subunit, thereby inhibiting IL-4 and IL-13 signaling and decreasing IgE production. A Phase 3 study (NCT04442269) is evaluating the efficacy and safety of dupilumab, in participants with $\mathrm{ABPA}$, in particular the annualized rate of exacerbations.

Table 5. Monoclonal antibodies for fungal respiratory infections.

\begin{tabular}{|c|c|c|c|c|c|c|c|}
\hline Indication & $\begin{array}{l}\text { Generic } \\
\text { Name }\end{array}$ & $\begin{array}{l}\text { Sponsoring } \\
\text { Company }\end{array}$ & Target & $\begin{array}{l}\text { Development } \\
\text { Stage }\end{array}$ & NCT & $\begin{array}{c}\text { Completion } \\
\text { Date }\end{array}$ & Status \\
\hline \multirow{2}{*}{$\begin{array}{l}\text { Aspergillus } \\
\text { fumigatus }\end{array}$} & Benralizumab & MedImmune & IL-5R $\alpha$ & Phase 4 & NCT04108962 & 2022 & Recruiting \\
\hline & Dupilumab & $\begin{array}{c}\text { Regeneron } \\
\text { Pharmaceuticals }\end{array}$ & IL-4/IL-13R & Phase 3 & NCT04442269 & 2023 & $\begin{array}{c}\text { Active, not } \\
\text { yet recruiting }\end{array}$ \\
\hline
\end{tabular}

IL, interleukin; IL-R: interleukinreceptor.

\subsection{Pharmacokinetics of Anti-Rti Abs}

\subsubsection{Administration Route}

Historically, Abs were mainly administered intravenously (IV). However, an increasing number of molecules have been developed for the subcutaneous (SC) or intramuscular (IM) routes, the latter being used for palivizumab and obiltoxaximab. Absorption following the SC or IM route occurs by convective transport via lymphatic system, leading to a time of peak blood concentration ranging from two to 10 days [56]. The distribution of an $\mathrm{Ab}$ is limited by their hydrophilicity and high molecular mass: its central and steady-state volumes of distribution are, respectively, 3-5 and 5-15 L. Elimination of Abs occurs via three main mechanisms: (i) endogenous catabolism; (ii) target-mediated elimination; and (iii) elimination mediated by ADA.

\subsubsection{Endogenous (Non-Specific) Catabolism}

After cellular uptake, IgGs bind to neonatal Fc receptor ( $\mathrm{FcRn})$ in acidic $\mathrm{pH}$ conditions. FcRnensures their protection from lysosomal degradation and recycling into the bloodstream, which explainsan IgG's long elimination (T1/2). Accordingly, most anti-infectious Abs - as they are IgGs - have a T1/2 of approximately 20 days. The increasing knowledge in $\mathrm{Ab}$ molecular biology has granted the emergence of $\mathrm{Ab}$ engineering with specific mutations that modulate the Ab's properties. In particular, mutations in the Fc portion were specifically designed to increase their affinity towards FcRn. This allowed for MEDI-4893 to have dose-proportional concentrations and to sustain serum exposure above the target level 30 days after infusion, which may provide a longer duration of disease prevention [73]. The administration of Abs in children has been a challenge, especially anti-RSV Abs in 
premature infants. In general, Ab elimination seems to increase with young age [20]. The reasons remain unclear, even if a possible explanation is a decreased FcRn expression in infants. This may explain the lower T1/2 of nirsevimabin infants than in adults $[26,74]$.

\subsubsection{Target-Mediated (Specific) Elimination}

Abs bind to their antigen target with a high affinity; the Ab-target complex is then eliminated by the immune system. Thus, Abs are drained by antigen targets. The targetmediated elimination rate is correlated to the target amount and turnover, and the elimination rate of the $\mathrm{Ab}$-target complexes. Target-mediated elimination results in nonlinear elimination decay, which further complicates the design of optimal dosing [75].

Target-mediated elimination is often reported in dose-ranging studies, where an increase of $\mathrm{T} 1 / 2$ with dose is observed, as for obiltoxaximab [58]. At the tested doses, almost all anti-infectious Abs presented linear elimination decay. However, the majority of PK studies were achieved in healthy subjects, who present null or low amounts of targets and therefore no target-mediated elimination. Extrapolating the PK findings in healthy subjects to define the dose and regimen in RTI patients may not be appropriate, since target-mediated elimination of Abs may be increased in infected patients, which may ultimately lead to underexposure and treatment failure.

\subsubsection{Elimination Mediated by Anti-Drug Antibody}

When investigated in Ab PK studies, the presence of ADA is mostly associated with increased $\mathrm{Ab}$ clearance, and thus decreased exposure that altogether can lead to loss of efficacy. The presence of ADA is observed in approximately 2-20\% of patients, variable with antibody and underlying disease [20], primarily with palivizumab [76] and nirsevimab [26].

\subsubsection{Pharmacokinetic Variability, Concentration-Response Relationship and} Optimal Dosing

The PK of anti-infectious $\mathrm{Ab}$ is variable among patients and may be influenced by several factors, including age, the presence of ADA and the amount of antigen targets (viruses, bacteria or target cells). This variability leads to a complex PK behavior for some Abs, which complicates the design of an optimal dose in therapeutics. In humans, a drug's PK is quantified using either non-compartmental methods or population compartmental modeling [77]. The latter one has been more and more used, because it allows sound quantification of PK, concentration-effect relationship variability and the design of an optimal dosing regimen. This approach is further needed in case of complex PK due to the influence of antigen burden. Up to date, PK results using this approach were reported only for palivizumab [76]. Overall, the PK of anti-infectious Abs needs further investigations; new clinical studies are needed to soundly quantify the dose-concentration-response relationship and to design optimal dosing strategies for anti-infectious Ab treatments.

\section{Discussion and Conclusions}

Antibiotic resistance has become epidemic with projections estimating mortality from bacterial infections to be around 10 million/year in 2050, surpassing combined deaths from cancer and heart diseases [78]. In particular, the ESKAPE pathogens, comprising four bacteria associated with RTIs (Staphylococcus aureus, Klebisella pneumoniae, Acinetobacter baumannii and Pseudomonas aeruginosa), represent a serious healthcare issue, requiring innovative therapeutic strategies. In this context, therapeutic Abs are a promising alternative to antibiotics for infectious diseases and they have already proven successful [14].

Since 2018, with the exception of the anti-SARS-CoV-2 Ab, the pipeline of anti-RTI Abs in clinical development has undergone a significant contraction: nine Abs have been discontinued and no novel $\mathrm{Ab}$ has entered into clinical evaluation [14]. This highlights that even if being a thriving focus of research, the development of anti-infectious Abs remains challenging. First, the selection of the antigenic target continues to be disputed. Indeed, many Abs have been designed to bind to epitopes that are not conserved or display strain heterogeneity (e.g., lipopolysaccharides (LPS), exopolysaccharides), questioning the clinical 
relevance of targeting a unique pathogenic antigen. This strategy is even more arguable for viral infections, considering the rapid evolution and selection of escape mutants, especially for emerging viruses [5]. In addition, extrapolated data from animal models are fraught with conjectures in proper target selection. For example, the development of an anti-S. aureus $\mathrm{Ab}$ has been hindered with the focus made on surface structure targeting (capsule, lipoteichoic acid) and the aim of opsonophagocytic killing, while neglecting the development of anti-toxin Abs. This was based on a misinterpretation of the toxins' importance in a mouse model, which are nearly insensitive to leukocidins [63,79]. In this context, refined preclinical models allowing a better understanding of the pathogenesis of lung infections and accelerated diagnostic procedures may be fundamental to improve the success of anti-RTI Abs.

Novel technologies-comprising multivalent antibodies, molecules derived from antibodies or novel formats towards antibody-drug conjugate (ADC) or antibody-antibiotic conjugate (AAC) [80] - may offer alternatives to overcome the antigenic variability of pathogens and resistance to standard treatments. Antibody mixtures, e.g., cocktails targeting different microbial pathogenic mechanisms, or Abs combined to standard-of-care antibiotics and immunomodulators, may help dealing with multidrug-resistant strains (MDR), while avoiding the appearance of cross-resistance and the disruption of the normal microflora of patients. Engineering strategies may also improve the Ab's PK and/or their effectors functions.

Another limitation of anti-RTI Ab development is related to their cost and their business environment. Besides the costs associated with the preclinical development of any drug, $\mathrm{Ab}$ production is expensive, evaluated to be several tens of thousands of dollars per $\mathrm{g}$ of material [81]. Business models and potential markets depend on the pathogen and may be more limited in RTI than inflammatory diseases or cancer. Moreover, development of anti-RTI Abs is hindered by uncertainties about (i) testing preventive or therapeutic settings in patients; (ii) definition of the best-suited population for the intervention; and (iii) the choice of appropriate end-points, especially when evaluating them in acute RTIs [82]. Altogether, these concerns make future economic returns unsure, which may possibly explain the discontinuation of several anti-RTI Ab programs. However, it would be reasonable to put the $\mathrm{Ab}$ cost in view of the average expense for the treatment of patients suffering from MDR lung infections, which is estimated to be increasingly high [78,83]. Solutions may come from innovations (i) in Ab production (plant cell-based or DNA plasmid-delivered muscle cell-based), which will improve yields; and (ii) in Ab delivery (considering novel intramuscular or inhalation routes), which will reduce therapeutic dosing while improving efficacy $[14,84]$ - both concurring to limit production costs.

Notably, the last few months has witnessed the fundamental place of Abs to fight emerging pathogens. Overall, 13 clinical trials evaluating Abs against SARS-CoV-2 are ongoing (1). Immunomodulating Abs, such as tocilizumab, already showed promising results, most likely preventing SARS-CoV-2 inflammatory-induced lung damage. This increasing global trend is evidence for a strong dynamic that could place therapeutic Abs as an effective complement to the conventional anti-RTI arsenal. Along with improvements in production and engineering, this should fuel the expansion of an Ab-based anti-RTI armamentarium. However, additional studies are required to decipher more clearly their PK, especially in diseased patients rather than in healthy subjects, and to investigate alternative delivery routes, like inhalation. All these issues represent substantial costs regarding preclinical and development processes, but the recent SARS-CoV-2 pandemic underscored how relevant and critical it is to promote alternative therapeutic strategies and anti-pathogen preparedness.

Author Contributions: A.M., A.C., G.D., T.S., and N.H.-V. participated in the review of research; T.S. prepared Figure 1; A.M. and A.C., prepared the tables; all authors contributed to the manuscript. All authors have read and agreed to the published version of the manuscript. 
Funding: The authors thank the laboratory of excellence Mab Improve (Tours-Montpellier, ANR10-LABX -53-01) and the Region Centre-Val de Loire (Novantinh and Infinhitim programs) for their support.

Institutional Review Board Statement: Not applicable.

Informed Consent Statement: Not applicable.

Data Availability Statement: Not applicable.

Conflicts of Interest: A.M., A.C., G.D., D.T. and T.S. do not declare conflicts of interest. N.H.-V. is co-founder and scientific expert for Cynbiose Respiratory; she receives consultancy fees from Argenx, Eli Lilly, and research support from Sanofi, Aerogen Ltd. and Signia Therapeutics. These companies had no role in review design, data collection and analysis, decision to publish, or preparation of the manuscript.

\section{References}

1. Foundation, W.L. Acute Respiratory Infections Atlas; World Lung Foundation: New York, NY, USA, 2010.

2. Schluger, N.W.; Koppaka, R. Lung disease in a global context. A call for public health action. Ann. Am. Thorac. Soc. 2014, 11, 407-416. [CrossRef]

3. Dagan, R.; Bhutta, Z.A.; de Quadros, C.A.; Garau, J.; Klugman, K.P.; Khuri-Bulos, N.; Levine, O.; Saha, S.K.; Sow, S.; Were, F.; et al. The remaining challenge of pneumonia: The leading killer of children. Pediatr. Infect. Dis. J. 2011, 30, 1-2. [CrossRef] [PubMed]

4. Liu, L.; Oza, S.; Hogan, D.; Chu, Y.; Perin, J.; Zhu, J.; Lawn, J.E.; Cousens, S.; Mathers, C.; Black, R.E. Global, regional, and national causes of under-5 mortality in 2000-15: An updated systematic analysis with implications for the Sustainable Development Goals. Lancet 2016, 388, 3027-3035. [CrossRef]

5. Salazar, G.; Zhang, N.; Fu, T.M.; An, Z. Antibody therapies for the prevention and treatment of viral infections. NPJ Vaccines 2017, 2, 19. [CrossRef] [PubMed]

6. Domenech, M.; Sempere, J.; de Miguel, S.; Yuste, J. Combination of Antibodies and Antibiotics as a Promising Strategy against Multidrug-Resistant Pathogens of the Respiratory Tract. Front. Immunol. 2018, 9, 2700. [CrossRef]

7. Desoubeaux, G.; Pelegrin, M. [Monoclonal antibodies in infectious diseases: New partners in the therapeutic arsenal]. Med. Sci. 2019, 35, 1008-1013. [CrossRef]

8. Desoubeaux, G.; Reichert, J.M.; Sleeman, M.; Reckamp, K.L.; Ryffel, B.; Adamczewski, J.P.; Sweeney, T.D.; Vanbever, R.; Diot, P.; Owen, C.A.; et al. Therapeutic monoclonal antibodies for respiratory diseases: Current challenges and perspectives, March 31-April 1, 2016, Tours, France. $m A$ bs 2016, 8, 999-1009. [CrossRef]

9. Desoubeaux, G.; Daguet, A.; Watier, H. Therapeutic antibodies and infectious diseases, Tours, France, November 20-22, 2012. $m A b s$ 2013, 5, 626-632. [CrossRef]

10. Cecil, R.L.; Larsen, N.P. Clinical and Bacteriologic Study of One Thousand Cases of Lobar Pneumonia with Special Reference to the Therapeutic Value of Pneumococcus Antibody Solution: Preliminary Report. JAMA 1922, 79, 343-349. [CrossRef]

11. Pelegrin, M.; Naranjo-Gomez, M.; Piechaczyk, M. Antiviral Monoclonal Antibodies: Can They Be More Than Simple Neutralizing Agents? Trends Microbiol. 2015, 23, 653-665. [CrossRef]

12. Wang, C.; Li, W.; Drabek, D.; Okba, N.M.A.; van Haperen, R.; Osterhaus, A.; van Kuppeveld, F.J.M.; Haagmans, B.L.; Grosveld, F.; Bosch, B.J. A human monoclonal antibody blocking SARS-CoV-2 infection. Nat. Commun. 2020, 11, 2251. [CrossRef]

13. Kohler, G.; Milstein, C. Continuous cultures of fused cells secreting antibody of predefined specificity. Nature 1975, 256, 495-497. [CrossRef]

14. Secher, T.; Guilleminault, L.; Reckamp, K.; Amanam, I.; Plantier, L.; Heuze-Vourc'h, N. Therapeutic antibodies: A new era in the treatment of respiratory diseases? Pharmacol. Ther. 2018, 189, 149-172. [CrossRef]

15. Glanville, J.; Zhai, W.; Berka, J.; Telman, D.; Huerta, G.; Mehta, G.R.; Ni, I.; Mei, L.; Sundar, P.D.; Day, G.M.; et al. Precise determination of the diversity of a combinatorial antibody library gives insight into the human immunoglobulin repertoire. Proc. Natl. Acad. Sci. USA 2009, 106, 20216-20221. [CrossRef]

16. Marks, J.D.; Hoogenboom, H.R.; Bonnert, T.P.; McCafferty, J.; Griffiths, A.D.; Winter, G. By-passing immunization. Human antibodies from V-gene libraries displayed on phage. J. Mol. Biol. 1991, 222, 581-597. [CrossRef]

17. Hey, A. History and Practice: Antibodies in Infectious Diseases. Microbiol. Spectr. 2015, 3, AID-0026-2014. [CrossRef] [PubMed]

18. Carter, P.J. Potent antibody therapeutics by design. Nat. Rev. Immunol. 2006, 6, 343-357. [CrossRef]

19. Irani, V.; Guy, A.J.; Andrew, D.; Beeson, J.G.; Ramsland, P.A.; Richards, J.S. Molecular properties of human IgG subclasses and their implications for designing therapeutic monoclonal antibodies against infectious diseases. Mol. Immunol. 2015, 67, 171-182. [CrossRef] [PubMed]

20. Bensalem, A.; Ternant, D. Pharmacokinetic Variability of Therapeutic Antibodies in Humans: A Comprehensive Review of Population Pharmacokinetic Modeling Publications. Clin. Pharmacokinet. 2020, 59, 857-874. [CrossRef]

21. Griffiths, C.; Drews, S.J.; Marchant, D.J. Respiratory Syncytial Virus: Infection, Detection, and New Options for Prevention and Treatment. Clin. Microbiol. Rev. 2017, 30, 277-319. [CrossRef] [PubMed] 
22. Meng, J.; Hotard, A.L.; Currier, M.G.; Lee, S.; Stobart, C.C.; Moore, M.L. Respiratory Syncytial Virus Attachment Glycoprotein Contribution to Infection Depends on the Specific Fusion Protein. J. Virol. 2016, 90, 245-253. [CrossRef] [PubMed]

23. Tang, A.; Chen, Z.; Cox, K.S.; Su, H.P.; Callahan, C.; Fridman, A.; Zhang, L.; Patel, S.B.; Cejas, P.J.; Swoyer, R.; et al. A potent broadly neutralizing human RSV antibody targets conserved site IV of the fusion glycoprotein. Nat. Commun. 2019, $10,4153$. [CrossRef] [PubMed]

24. American Academy of Pediatrics Subcommittee on Diagnosis and Management of Bronchiolitis. Diagnosis and management of bronchiolitis. Pediatrics 2006, 118, 1774-1793. [CrossRef]

25. Nair, H.; Nokes, D.J.; Gessner, B.D.; Dherani, M.; Madhi, S.A.; Singleton, R.J.; O’Brien, K.L.; Roca, A.; Wright, P.F.; Bruce, N.; et al. Global burden of acute lower respiratory infections due to respiratory syncytial virus in young children: A systematic review and meta-analysis. Lancet 2010, 375, 1545-1555. [CrossRef]

26. Domachowske, J.B.; Khan, A.A.; Esser, M.T.; Jensen, K.; Takas, T.; Villafana, T.; Dubovsky, F.; Griffin, M.P. Safety, Tolerability and Pharmacokinetics of MEDI8897, an Extended Half-life Single-dose Respiratory Syncytial Virus Prefusion F-targeting Monoclonal Antibody Administered as a Single Dose to Healthy Preterm Infants. Pediatric Infect. Dis. J. 2018, 37, 886-892. [CrossRef]

27. Maas, B.; Aliprantis, A.; Wolford, D.; Fayad, G.; Vora, K.; Geng, D.; Ma, H.; Caro, L. RSV Monoclonal Antibody (MK-1654) Phase 1 Pharmacokinetics (PK) in Healthy Adults and Population PK Modeling to Support Pediatric Development. Open Forum Infect. Dis. 2018, 5, S424-S425. [CrossRef]

28. Fortes, H.R.; von Ranke, F.M.; Escuissato, D.L.; Araujo Neto, C.A.; Zanetti, G.; Hochhegger, B.; Souza, C.A.; Marchiori, E. Recurrent respiratory papillomatosis: A state-of-the-art review. Respir. Med. 2017, 126, 116-121. [CrossRef] [PubMed]

29. Ahn, J.; Bishop, J.A.; Roden, R.B.S.; Allen, C.T.; Best, S.R.A. The PD-1 and PD-L1 pathway in recurrent respiratory papillomatosis. Laryngoscope 2018, 128, E27-E32. [CrossRef]

30. Boyerinas, B.; Jochems, C.; Fantini, M.; Heery, C.R.; Gulley, J.L.; Tsang, K.Y.; Schlom, J. Antibody-Dependent Cellular Cytotoxicity Activity of a Novel Anti-PD-L1 Antibody Avelumab (MSB0010718C) on Human Tumor Cells. Cancer Immunol. Res. 2015, 3, 1148-1157. [CrossRef]

31. Allen, C.T.; Lee, S.; Norberg, S.M.; Kovalovsky, D.; Ye, H.; Clavijo, P.E.; Hu-Lieskovan, S.; Schlegel, R.; Schlom, J.; Strauss, J.; et al. Safety and clinical activity of PD-L1 blockade in patients with aggressive recurrent respiratory papillomatosis. J. Immunother. Cancer 2019, 7, 119. [CrossRef]

32. Tumeh, P.C.; Harview, C.L.; Yearley, J.H.; Shintaku, I.P.; Taylor, E.J.; Robert, L.; Chmielowski, B.; Spasic, M.; Henry, G.; Ciobanu, V.; et al. PD-1 blockade induces responses by inhibiting adaptive immune resistance. Nature 2014, 515, 568-571. [CrossRef]

33. World Health Organization. Global Influenza Strategy 2019-2030. Available online: https://www.who.int/influenza/global_ influenza_strategy_2019_2030/en/(accessed on 30 October 2020).

34. Zhou, F.; Trieu, M.C.; Davies, R.; Cox, R.J. Improving influenza vaccines: Challenges to effective implementation. Curr. Opin. Immunol. 2018, 53, 88-95. [CrossRef] [PubMed]

35. Toots, M.; Plemper, R.K. Next-generation direct-acting influenza therapeutics. Transl. Res. J. Lab. Clin. Med. 2020, $220,33-42$. [CrossRef]

36. Wollacott, A.M.; Boni, M.F.; Szretter, K.J.; Sloan, S.E.; Yousofshahi, M.; Viswanathan, K.; Bedard, S.; Hay, C.A.; Smith, P.F.; Shriver, Z.; et al. Safety and Upper Respiratory Pharmacokinetics of the Hemagglutinin Stalk-Binding Antibody VIS410 Support Treatment and Prophylaxis Based on Population Modeling of Seasonal Influenza A Outbreaks. EBioMedicine 2016, 5, 147-155. [CrossRef] [PubMed]

37. Hershberger, E.; Sloan, S.; Narayan, K.; Hay, C.A.; Smith, P.; Engler, F.; Jeeninga, R.; Smits, S.; Trevejo, J.; Shriver, Z.; et al. Safety and efficacy of monoclonal antibody VIS410 in adults with uncomplicated influenza A infection: Results from a randomized, double-blind, phase-2, placebo-controlled study. EBioMedicine 2019, 40, 574-582. [CrossRef] [PubMed]

38. Sedeyn, K.; Saelens, X. New antibody-based prevention and treatment options for influenza. Antivir. Res. 2019, $170,104562$. [CrossRef]

39. Oldach, D.; Narayan, K.; Schaefers, K.; Sloan, S.; Smith, P.; Bliss, R.; Arbrough, J.; Shriver, Z. A global, randomised, dou-ble-blind, placebo-controlled study evaluating safety and efficacy of vis410 in combination with oseltamivir versus oselta-mivir alone in hospitalized adults with influenza a requiring oxygen. In Option X for the Control of Influenza; International Society for Influenza and other Respiratory Virus Diseases (ISIRV): Singapore, 2019; Volume 11754.

40. Park, M.D. Macrophages: A Trojan horse in COVID-19? Nat. Rev. Immunol. 2020, 20, 351. [CrossRef]

41. Polidoro, R.B.; Hagan, R.S.; de Santis Santiago, R.; Schmidt, N.W. Overview: Systemic Inflammatory Response Derived from Lung Injury Caused by SARS-CoV-2 Infection Explains Severe Outcomes in COVID-19. Front. Immunol. 2020, 11, 1626. [CrossRef]

42. Sariol, A.; Perlman, S. Lessons for COVID-19 Immunity from Other Coronavirus Infections. Immunity 2020. [CrossRef]

43. Blanco-Melo, D.; Nilsson-Payant, B.E.; Liu, W.C.; Uhl, S.; Hoagland, D.; Moller, R.; Jordan, T.X.; Oishi, K.; Panis, M.; Sachs, D.; et al. Imbalanced Host Response to SARS-CoV-2 Drives Development of COVID-19. Cell 2020, 181, 1036-1045 e1039. [CrossRef]

44. Lega, S.; Naviglio, S.; Volpi, S.; Tommasini, A. Recent Insight into SARS-CoV2 Immunopathology and Rationale for Potential Treatment and Preventive Strategies in COVID-19. Vaccines 2020, 8, 224. [CrossRef]

45. Poduri, R.; Joshi, G.; Jagadeesh, G. Drugs targeting various stages of the SARS-CoV-2 life cycle: Exploring promising drugs for the treatment of Covid-19. Cell. Signal. 2020. [CrossRef] 
46. Guarali, G.; Meschiari, M.; Cozzi-Lepri, A.; Milic, J.; Tonelli, R.; Menozzi, M.; Franceschini, E.; Cuomo, G.; Orlando, G.; Borghi, V.; et al. Tocilizumab in patients with severe COVID-19: A retrospective cohort study. Lancet Rheumatol. 2020, 2, 474-484. [CrossRef]

47. Gritti, G.; Raimondi, F.; Ripamonti, D.; Riva, I.; Landi, F.; Alborghetti, L.; Frigeni, M.; Damiani, M.; Micò, C.; Fagiuoli, S.; et al. IL-6 signalling pathway inactivation with siltuximab in patients with COVID-19 respiratory failure: An observational cohort study. medRxiv 2020. [CrossRef]

48. U.S. Food and Drug Administration. Emergency Use Authorization. Available online: https://www.fda.gov/emergencypreparedness-and-response/mcm-legal-regulatory-and-policy-framework/emergency-use-authorization\#covid19euas (accessed on 12 January 2021).

49. Dasaraju, P.V.; Liu, C. Infections of the respiratory system. In Medical Microbiology, 4th ed.; Baron, S., Ed.; University of Texas Medical Branch at Gavelston: Gavelston, TX, USA, 1996.

50. GBD Lower Respiratory Infections Collaborators. Estimates of the global, regional, and national morbidity, mortality, and aetiologies of lower respiratory infections in 195 countries, 1990-2016: A systematic analysis for the Global Burden of Disease Study 2016. Lancet Infect. Dis. 2018, 18, 1191-1210. [CrossRef]

51. Mackenzie, G. The definition and classification of pneumonia. Pneumonia 2016, 8, 14. [CrossRef] [PubMed]

52. Van der Poll, T.; Opal, S.M. Pathogenesis, treatment, and prevention of pneumococcal pneumonia. Lancet 2009, 374, 1543-1556. [CrossRef]

53. Hendricks, K.; Vieira, A.R.; Marston, C.K. Anthrax. Available online: https:/ / wwwnc.cdc.gov (accessed on 30 October 2020).

54. Frankel, A.E.; Kuo, S.R.; Dostal, D.; Watson, L.; Duesbery, N.S.; Cheng, C.P.; Cheng, H.J.; Leppla, S.H. Pathophysiology of anthrax. Front. Biosci. 2009, 14, 4516-4524. [CrossRef]

55. Casadevall, A. Antibodies for defense against biological attack. Nat. Biotechnol. 2002, 20, 114. [CrossRef]

56. Migone, T.S.; Subramanian, G.M.; Zhong, J.; Healey, L.M.; Corey, A.; Devalaraja, M.; Lo, L.; Ullrich, S.; Zimmerman, J.; Chen, A.; et al. Raxibacumab for the treatment of inhalational anthrax. N. Engl. J. Med. 2009, 361, 135-144. [CrossRef]

57. Yamamoto, B.J.; Shadiack, A.M.; Carpenter, S.; Sanford, D.; Henning, L.N.; Gonzales, N.; O'Connor, E.; Casey, L.S.; Serbina, N.V. Obiltoxaximab Prevents Disseminated Bacillus anthracis Infection and Improves Survival during Pre- and Postexposure Prophylaxis in Animal Models of Inhalational Anthrax. Antimicrob. Agents Chemother. 2016, 60, 5796-5805. [CrossRef] [PubMed]

58. Nagy, C.F.; Leach, T.S.; King, A.; Guttendorf, R. Safety, Pharmacokinetics, and Immunogenicity of Obiltoxaximab After Intramuscular Administration to Healthy Humans. Clin. Pharmacol. Drug Dev. 2018, 7, 652-660. [CrossRef] [PubMed]

59. Tong, S.Y.; Davis, J.S.; Eichenberger, E.; Holland, T.L.; Fowler, V.G., Jr. Staphylococcus aureus infections: Epidemiology, pathophysiology, clinical manifestations, and management. Clin. Microbiol. Rev. 2015, 28, 603-661. [CrossRef] [PubMed]

60. Grgurich, P.E.; Hudcova, J.; Lei, Y.; Sarwar, A.; Craven, D.E. Management and prevention of ventilator-associated pneumonia caused by multidrug-resistant pathogens. Expert Rev. Respir. Med. 2012, 6, 533-555. [CrossRef]

61. Reyes-Robles, T.; Torres, V.J. Staphylococcus aureus Pore-Forming Toxins. Clin. Microbiol. Rev. 2017, 409, 121-144. [CrossRef]

62. Spaan, A.N.; van Strijp, J.A.G.; Torres, V.J. Leukocidins: Staphylococcal bi-component pore-forming toxins find their receptors. Nat. Rev. Microbiol. 2017, 15, 435-447. [CrossRef]

63. Diep, B.A.; Hilliard, J.J.; Le, V.T.; Tkaczyk, C.; Le, H.N.; Tran, V.G.; Rao, R.L.; Dip, E.C.; Pereira-Franchi, E.P.; Cha, P.; et al. Targeting Alpha Toxin To Mitigate Its Lethal Toxicity in Ferret and Rabbit Models of Staphylococcus aureus Necrotizing Pneumonia. Antimicrob. Agents Chemother. 2017, 61. [CrossRef]

64. Hua, L.; Cohen, T.S.; Shi, Y.; Datta, V.; Hilliard, J.J.; Tkaczyk, C.; Suzich, J.; Stover, C.K.; Sellman, B.R. MEDI4893* Promotes Survival and Extends the Antibiotic Treatment Window in a Staphylococcus aureus Immunocompromised Pneumonia Model. Antimicrob. Agents Chemother. 2015, 59, 4526-4532. [CrossRef]

65. Hilliard, J.J.; Datta, V.; Tkaczyk, C.; Hamilton, M.; Sadowska, A.; Jones-Nelson, O.; O’Day, T.; Weiss, W.J.; Szarka, S.; Nguyen, V.; et al. Anti-alpha-toxin monoclonal antibody and antibiotic combination therapy improves disease outcome and accelerates healing in a Staphylococcus aureus dermonecrosis model. Antimicrob. Agents Chemother. 2015, 59, 299-309. [CrossRef] [PubMed]

66. François, B.; Sanchez, M.G.; Eggimann, P.; Dequin, P.; Laterre, P.; Huberlant, V.; Escudero, D.; Boulain, T.; Bretonniere, C.; Pugin, J.; et al. Suvratoxumab Reduces Staphylococcus Aureus Pneumonia in High-Risk ICU Patients: Results of the SAATELLITE Study. In Proceedings of the American Thoracic Society 2019 International Conference, Dallas, TX, USA, 17-22 May 2019.

67. Drumm, J.E.; Mielach, A.; Aridis Pharmaceuticals, Inc. Presents Positive Phase 2a Safety and Efficacy Data of Salvecin ${ }^{\mathrm{TM}}$ (AR-301) in Patients with Severe Pneumonia Caused by Staphylococcus aureus During the 2017 ASM Microbe Congress. Newswire, P., Ed.; 2017. Available online: https:/ / www.prnewswire.com/ (accessed on 13 February 2021).

68. Sadikot, R.T.; Blackwell, T.S.; Christman, J.W.; Prince, A.S. Pathogen-host interactions in Pseudomonas aeruginosa pneumonia. Am. J. Respir. Crit. Care Med. 2005, 171, 1209-1223. [CrossRef]

69. Que, Y.A.; Lazar, H.; Wolff, M.; Francois, B.; Laterre, P.F.; Mercier, E.; Garbino, J.; Pagani, J.L.; Revelly, J.P.; Mus, E.; et al. Assessment of panobacumab as adjunctive immunotherapy for the treatment of nosocomial Pseudomonas aeruginosa pneumonia. Eur. J. Clin. Microbiol. Infect. Dis. 2014, 33, 1861-1867. [CrossRef]

70. Ryder, C.; Byrd, M.; Wozniak, D.J. Role of polysaccharides in Pseudomonas aeruginosa biofilm development. Current Opin. Microbiol. 2007, 10, 644-648. [CrossRef] [PubMed] 
71. Greenberger, P.A.; Bush, R.K.; Demain, J.G.; Luong, A.; Slavin, R.G.; Knutsen, A.P. Allergic bronchopulmonary aspergillosis. J. Allergy Clin. Immunol. Pract. 2014, 2, 703-708. [CrossRef]

72. Soeda, S.; Kono, Y.; Tsuzuki, R.; Yamawaki, S.; Katsube, O.; To, M.; To, Y. Allergic bronchopulmonary aspergillosis successfully treated with benralizumab. J. Allergy Clin. Immunol. Pract. 2019, 7, 1633-1635. [CrossRef] [PubMed]

73. Khan, A.; Yu, X.Q.; François, B.; Eggimann, P.; Huberlant, V.; Dequin, P.F.; Valia, J.C.; Ali, S.O.; Jensen, K.; Colbert, S.; et al. Interim pharmacokinetic analysis from the SAATELLITE Phase 2 Clinical Trial of Suvratoxumab (MEDI4893), an extended half-life monoclonal antibody against Staphylococcus aureus alpha toxin. In Proceedings of the 28th ECCMID, Madrid, Spain, 21-24 April 2018.

74. Griffin, M.P.; Khan, A.A.; Esser, M.T.; Jensen, K.; Takas, T.; Kankam, M.K.; Villafana, T.; Dubovsky, F. Safety, Tolerability, and Pharmacokinetics of MEDI8897, the Respiratory Syncytial Virus Prefusion F-Targeting Monoclonal Antibody with an Extended Half-Life, in Healthy Adults. Antimicrob. Agents Chemother. 2017, 61. [CrossRef]

75. Ternant, D.; Azzopardi, N.; Raoul, W.; Bejan-Angoulvant, T.; Paintaud, G. Influence of Antigen Mass on the Pharmacokinetics of Therapeutic Antibodies in Humans. Clin. Pharmacokinet. 2019, 58, 169-187. [CrossRef] [PubMed]

76. Robbie, G.J.; Zhao, L.; Mondick, J.; Losonsky, G.; Roskos, L.K. Population pharmacokinetics of palivizumab, a humanized anti-respiratory syncytial virus monoclonal antibody, in adults and children. Antimicrob. Agents Chemother. 2012, 56, 4927-4936. [CrossRef]

77. Mould, D.R.; Upton, R.N. Basic concepts in population modeling, simulation, and model-based drug development-part 2: Introduction to pharmacokinetic modeling methods. CPT Pharmacomet. Syst. Pharmacol. 2013, 2, e38. [CrossRef]

78. O'Neill, J. Tackling Drug-Resistant Infections Globally: Final Report and Recommendations; Wellcome Trust UK Department of Health: London, UK, 2016.

79. Alonzo, F., 3rd; Benson, M.A.; Chen, J.; Novick, R.P.; Shopsin, B.; Torres, V.J. Staphylococcus aureus leucocidin ED contributes to systemic infection by targeting neutrophils and promoting bacterial growth in vivo. Mol. Microbiol. 2012, 83, 423-435. [CrossRef]

80. DiGiandomenico, A.; Sellman, B.R. Antibacterial monoclonal antibodies: The next generation? Curr. Opin. Microbiol. 2015, 27, 78-85. [CrossRef]

81. Buyel, J.F.; Twyman, R.M.; Fischer, R. Very-large-scale production of antibodies in plants: The biologization of manufacturing. Biotechnol. Adv. 2017, 35, 458-465. [CrossRef] [PubMed]

82. Sparrow, E.; Friede, M.; Sheikh, M.; Torvaldsen, S. Therapeutic antibodies for infectious diseases. Bull. World Health Organ. 2017, 95, 235-237. [CrossRef]

83. Simon, M.S.; Sfeir, M.M.; Calfee, D.P.; Satlin, M.J. Cost-effectiveness of ceftazidime-avibactam for treatment of carbapenemresistant Enterobacteriaceae bacteremia and pneumonia. Antimicrob. Agents Chemother. 2019, 63, e00897-19. [CrossRef] [PubMed]

84. Secher, T.; Dalonneau, E.; Ferreira, M.; Parent, C.; Azzopardi, N.; Paintaud, G.; Si-Tahar, M.; Heuze-Vourc'h, N. In a murine model of acute lung infection, airway administration of a therapeutic antibody confers greater protection than parenteral administration. J. Control. Release 2019, 303, 24-33. [CrossRef] [PubMed] 